\title{
MODELING AND ANALYSIS OF AN AUCTION-BASED LOGISTICS MARKET
}

\author{
Semra Ağralı \\ Dept. of Industrial and Systems Eng. \\ University of Florida, \\ 303 Weil Hall, Gainesville \\ FL 32611, USA \\ sagrali@ufl.edu
}

\author{
Barış Tan \\ Graduate School of Business \\ Koç University \\ Rumeli Feneri Yolu,34450 \\ Istanbul, Turkey \\ btan@ku.edu.tr
}

\author{
Fikri Karaesmen
}

Dept. of Industrial Engineering

Koç University

Rumeli Feneri Yolu,34450

Istanbul, Turkey

fkaraesmen@,ku.edu.tr

June 2007

\begin{abstract}
We consider a logistics spot market where the transportation orders from a number of firms are matched with two types of carriers through a reverse auction. In the spot market, local carriers compete with in-transit carriers that have lower costs. In order to analyze the effects of implementing a logistics spot market on these three parties: firms, local carriers, and in-transit carriers and also the effects of various system parameters, we develop a two-stage stochastic model. We first model the auction in a static setting and determine the expected auction price based on the number of carriers engaging in the auction and their cost distributions. We then develop a continuous-time Markov chain model to evaluate the performance of the system in a dynamic setting with random arrivals and possible abandonment of orders and carriers. By combining these two models, we evaluate the performance measures such as the expected auction price, price paid to the carriers, distribution of orders between local and in-transit carriers, and expected number of carriers and orders waiting at the logistics center in the long run. We present analytical and computational results related to the performance of the system and discuss operation of such a logistics spot market in Turkey.
\end{abstract}

Key Words: Logistics, Markov Chain Models, Performance Evaluation, Reverse Auctions

\section{Introduction}

We consider a logistics spot market where the transportation orders from a number of firms are matched with two types of carriers, local carriers and in-transit carriers with lower costs, through a reverse auction. Implementing such a logistics spot market has different effects on the three parties involved: firms, local carriers, and in-transit carriers. Since a reverse auction increases competition among carriers, it is expected that firms will realize lower transportation costs. Similarly, in-transit carriers benefit from the spot market since they get more orders with their lower prices. However, local carriers can be affected negatively, since they may lose some of their business to in-transit carriers. Although it is possible to foresee how each party is affected, deciding on implementation of a logistics spot market requires quantifying these effects. In other words, it is important to determine bow much and under which conditions each party gains or loses. Our objective in this study is to develop a simplified analytical model that captures important characteristics of a logistics spot market. This model can be used to quantify the effects of using an auction-based logistics spot market on firms, local carriers and in-transit carriers and also to analyze the effects of system parameters on the performance of the logistics market.

Different forms of marketplaces, including clearing houses, auction houses, and freight exchanges, are used in freight transportation (Nandiraju and Regan, 2003). This study is motivated by a logistics spot 
market, ESO Logistics Center (www.esolojistik.com) established in Eskişehir, Turkey. With the objective of lowering the transportation costs of the firms located in the Organized Industrial Zone, the Eskişehir Chamber of Industry (ESO) established a logistics center in 2003. The location of the ESO logistics center and the cities it serves are given Figure 1.

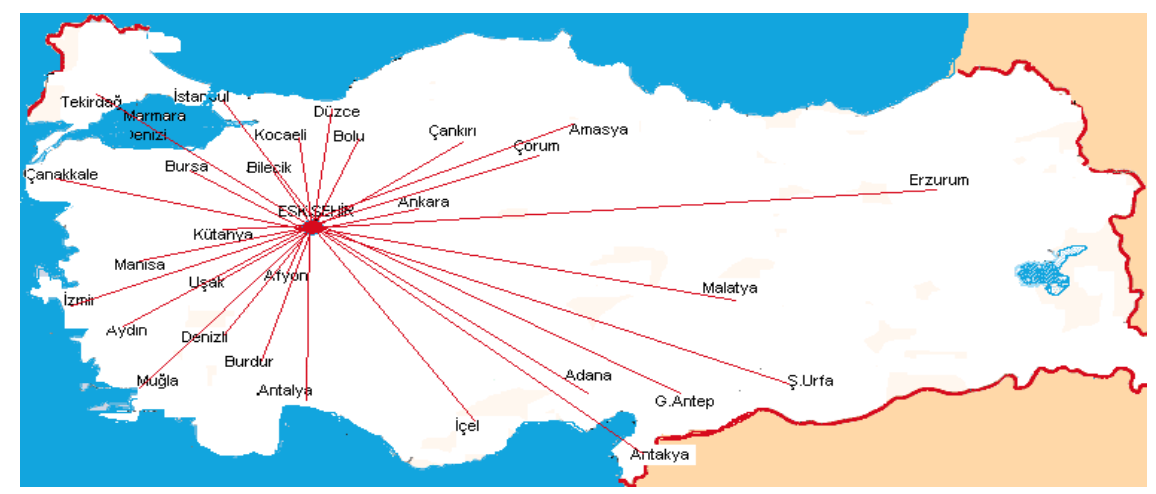

Figure 1. The transportation destinations (major Turkish cities) from Eskişehir

The logistics center attracts both local carriers and in-transit carriers that have delivered their loads and are returning to their bases. Since these in-transit carriers are often paid for both ways, their prices are much lower compared to the local carriers.

ESO reports that using a reverse auction mechanism to match orders from different firms and carriers lowered the transportation costs of the companies in the industrial zone around $30 \%$ in a short period. Our analysis of the ESO database, which includes detailed information on the auctions that took place between December 2003, when the system started its operation, and May 2005, confirms the savings. A summary of the available information in the database is given in Table 9 in the Appendix.

More specifically, when we compared the prices realized at the ESO Logistics Center with the market price and also with the fuel cost of a truck, we observed that the average transportation price that is realized in ESO Logistics Center is very close to the fuel price and is much lower than the market price as shown in Figure 2. In this analysis, the market price is the list price of transportation between Eskişehir and different destinations. This list is published by the Eskişehir Carriers' Cooperation. We also calculate the fuel cost by using the distance between Eskişehir and different destinations and fuel consumption rate of a typical truck used in transportation.

Since ESO expects that the number of firms using the Logistics Center will increase rapidly, a number of questions regarding how firms, local carriers, and in-transit carriers will be affected arise. For example, since local carriers have long-term relationship with ESO and also with the firms located in the industrial zone, it is important to quantify how much local carriers may lose and how much firms gain when intransit carriers are allowed in the logistics center. It is also of interest to understand how the balance between the arrival and abandonment of orders and carriers affects the average transportation prices. This study is motivated by the need of developing an analytical model to measure the performance of an auction-based logistics spot market with two-types of carriers. The model developed in this study allows us to answer questions similar to the ones given above. 


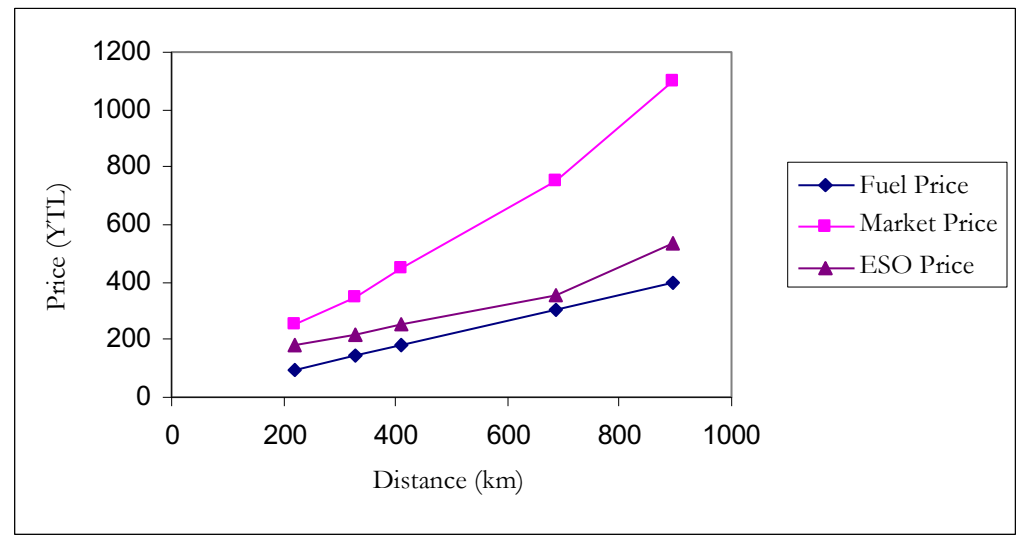

Figure 2. Fuel price, market price and the average transportation price realized at the ESO Logistics Center according to distance

In order to model and analyze the system, we present a general two-stage stochastic model of a logistics spot market with two types of carriers. In the first stage, an auction is analyzed in a static setting and the expected auction price is determined based on the number of local carriers and in-transit carriers and their cost distributions. In the second stage, we develop a continuous-time Markov Chain (CTMC) model to incorporate random arrivals of orders, local carriers, and in-transit carriers, and also possible abandonment of orders and carriers. By combining these two models, we evaluate performance measures such as the expected transportation price, the expected price paid to the carriers, distribution of orders between local and in-transit carrier, the expected number of carriers and orders waiting at the logistics center in the steady state. We also provide analytical results that show how the performance measures are affected by the system parameters.

The organization of the remaining part of the paper is as follows: the pertinent literature is reviewed in Section 2. Section 3 presents the general model and its assumptions. The methodology used in the analysis is given in Section 4. A number of performance measures are derived in Section 5. Section 6 presents analytical and numerical results related to the effects of system parameters on the performance measures. Finally, conclusions are given in Section 7.

\section{Literature Review}

In recent years, a number of studies that investigate using auctions in supply chains appeared in the literature. We limit our discussion to the papers that discuss auctions in logistics services procurements and also performance evaluation of auction-based systems. For a thorough review of auction theory, the reader is referred to the review of Klemperer (1999). Elmaghraby (2000) provides an overview of the contract competition and sourcing strategies that are studied in operations research and economics literature. Similarly, Chen et al. (2005) discuss using auctions for supply chain procurement. In addition to matching supply and demand efficiently, auctions can also be used to form a collaborative network among a number of truckload carriers (Song and Regan, 2003). 
In the supply chain literature, there are numerous studies on long term contracts between buyers and suppliers. For example, Ledyard et. al. (2002) discuss an auction used at Sears for a three year truckload carrier services contract. Alp et al. (2003) discuss a contract designed by a manufacturer to outsource its transportation requirements through a bidding process between competing carriers. However, our focus is on an auction-based logistics market where a short term contract, or a spot contract, is made between firms and carriers.

In addition to these long-term contracts, marketplaces such as clearing houses, auction houses, and freight exchanges facilitate efficient matching of supply and demand between shippers and carriers (Nandiraju and Regan, 2003). For example, Qi and Pich (2002) present a neutral exchange in the container industry that is based on matching bid and ask prices from multiple buyers and multiple sellers.

The number of studies that focus on the performance evaluation of systems that utilize auctions is limited. Veeramani and Wang (1997) present a queuing network model to evaluate the performance of auction-based distributed shop-floor control schemes. In a similar setting, Nandula and Dutta (2000) use Petri nets to evaluate the performance of a manufacturing system that uses auctions as a control strategy.

The number of studies on the stochastic analysis of the dynamics in an auction-based market is also limited. Our approach is similar to the works of Segev et al. (2001) and Vakrat and Seidmann (2000). Segev et al. (2001) model an online auction as a Markov Chain on a state space defined by the current price of the item and the number of buyers who are previously eliminated. By using this Markov Chain model, they determine a number of performance measures and validate their findings with real online auction data. Vakrat and Seidmann (2000) analyze the implications of the bidder's arrival process in an online auction by using a queueing-based model. On the modeling side, although we use a queueing-based approach as in the models of internet auctions, our situation is complicated by multiple carrier types and abandonment of orders and carriers in a logistics setting.

One of the main contributions of this paper is modeling and analysis of an auction-based logistics market where a short term contract, i.e. a spot contract, is made between shippers and two types of carriers. To our knowledge, this is the first study that models an auction-based logistics spot market with multiple types of carriers in a stochastic and dynamic setting. Furthermore, we show a number of structural results related to the effects of system parameters on the performance measures. More specifically, we analytically show how a number of performance measures are affected as the arrivaldeparture rates change. This approach can also be applied to other auctions in a dynamic setting.

\section{Model of the Logistics Center}

\subsection{Problem Description}

We consider a Logistics Center (LC) where multiple firms that want their goods to be transported to different destinations use auctions to make short-term contracts with carriers. At the LC there are multiple carriers that respond to transportation orders given by the shipper. When the shipper has a transportation order, she opens an auction by using an electronic reverse auction platform, i.e. a web-page designed for 
this process. All the carriers that would like to participate in the auction observe the order and submit their bids for that order. The order is given to the carrier who submitted the lowest bid. If no carriers are available or no bids are submitted, the company may cancel its auction, and either sends its goods by a logistics service provider or reopens the auction at a later time. Moreover, carriers who stop at the logistics center may abandon after some time if they cannot get an order.

Our objective is to determine the effects of using this logistics market on three parties involved: shippers, local carriers, and in-transit carriers by using an analytical model. This model also allows us to investigate the effects of various system parameters on the performance of the logistics spot market.

\subsection{Model Assumptions}

Orders. It is assumed that orders arrive randomly to the LC according to a Poisson process with rate $\lambda_{0}$. $N_{0}(t)$ and $N_{0}$ denote the number of orders available at the LC at time $t$ and in the steady state respectively. If there are no carriers available when an order arrives, the order can abandon after waiting some time. We assume that this time is exponentially distributed with rate $\varphi_{0}$.

The observed order inter-arrival distribution at the ESO logistics center that is depicted in Figure 3 supports the Poisson assumption for order arrivals. However, one can argue that the abandonment times of orders are not necessarily exponential. Our simulation experiments show that the first order performance measures that are of interest are not sensitive to the distribution of the abandonment times. Note that the same CTMC framework can be used to analyze abandonment times that have phase-type distributions.

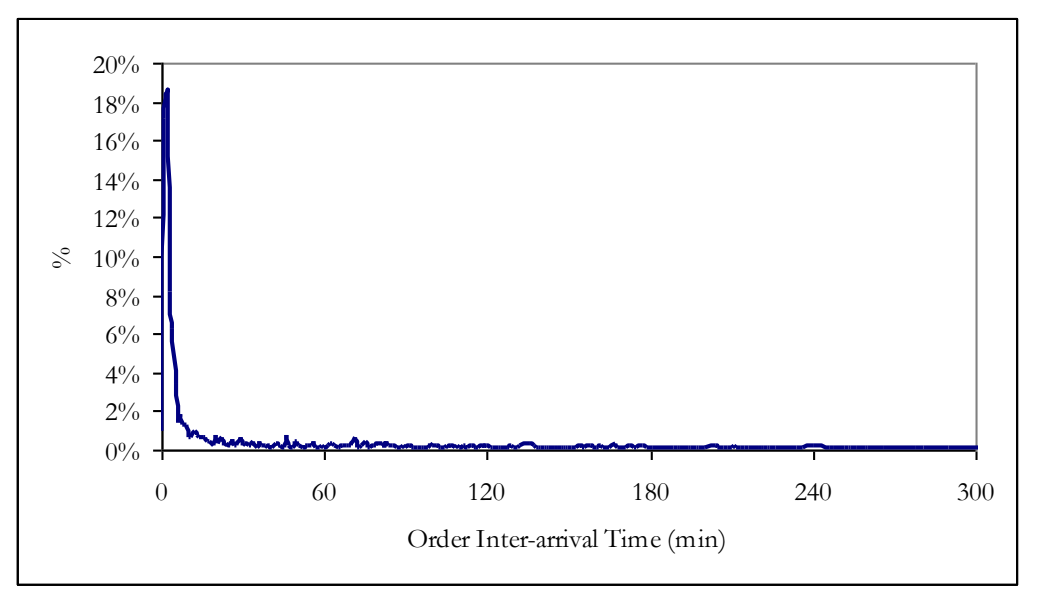

Figure 3. Inter-arrival time distribution of the orders received by the ESO Logistics Center during December 2003-March 2004

Carriers. We assume that there are two types of carriers, type 1 refers to local carriers that are based at the same region as the LC and type 2 refers to in-transit carriers that stop by the LC while traveling to their bases. $N_{i}(t)$ and $N_{i}$ denote the number of Type $i$ carriers at time $t$ and the number of Type $i$ carriers in steady state respectively, $i=1,2$.

Type $i$ carriers are assumed to arrive randomly to the logistics center according to a Poisson process with rate $\lambda_{i}, i=1,2 \quad$ A carrier can abandon the LC after waiting some time for receiving an order. We 
assume that this time is an exponentially distributed random variable with rate $\varphi_{i}$ for Type $i$ carriers. Figure 4 shows the distribution of the inter-arrival times of the bids placed for the orders received by the ESO Logistics Center.

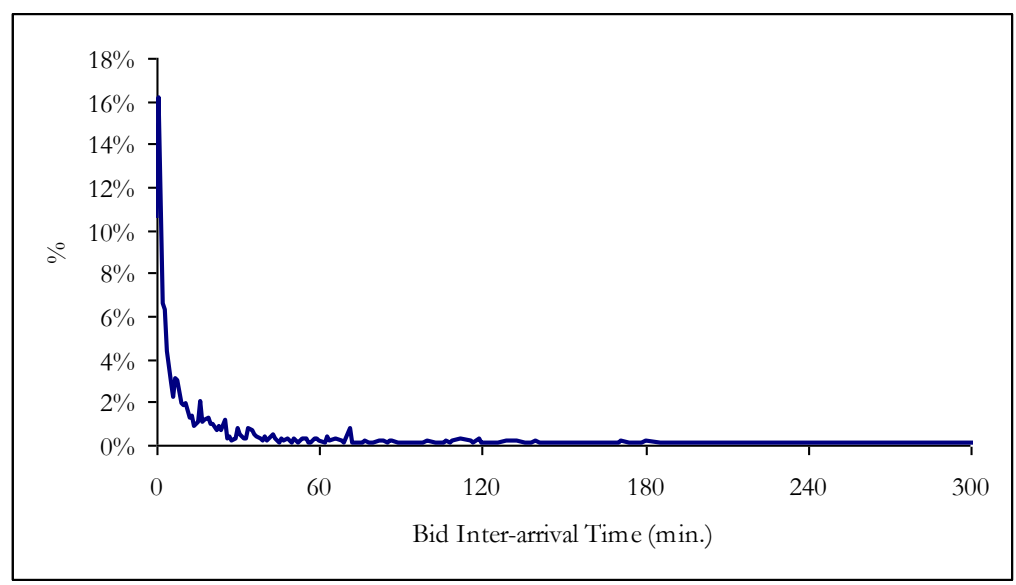

Figure 4. Inter-arrival time distribution of the bids placed for the orders received by the ESO Logistics Center during December 2003-March 2004

A Type $i$ carrier has a transportation cost of $C_{i}$ known only to him, which is considered by everyone to have been drawn from a distribution with cumulative density function (cdf) of $F_{i}(x), x \in\left[c_{i}, c_{i}^{\prime}\right]$ with expectation $\overline{c_{i}}$ for $i=1,2$. A transportation cost is determined for a specific destination and for a specific carrier. Therefore $F_{i}$ incorporates the variability in transportation costs for various destination-carrier couples.

In-transit carriers usually stop at the spot market on their way back to their home city after delivering an order and they are already paid for the remaining portion of their trip. Therefore, they are willing to accept much lower prices compared to local carriers to get an additional order that will increase their revenue for the same trip. Accordingly, we consider the case where the transportation costs of type 2 carriers are always lower than of type 1 carriers, i.e., $c_{1}>c_{2}^{\prime}$. It is possible to use the same methodology utilized in this paper to extend the results to overlapping cost distributions.

The carriers are assumed to be risk-neutral and they all maximize their own expected profit. In addition, it is assumed that there is no collusion between carriers.

Auction. A single-unit second-price sealed-bid auction, or the Vickrey auction, is used to decide which carrier gets an order of transporting one full-truck load. An order can not be split among different carriers. According to the Vickrey auction, the carrier with the lowest bid among all the bidding carriers at the LC gets the order and is paid the second-lowest price.

Figures 5 and 6 depict the histograms of the bids given to the orders from Eskişehir to Adana and the prices that these orders are taken, i.e. the winning bids, at the ESO Logistics Center. The wide range of the transportation prices to the same destination is a result of the operation of the spot market with intransit and local carriers. That is, lower prices are given by in-transit carriers and higher values are realized when there are a few carriers at the center. 


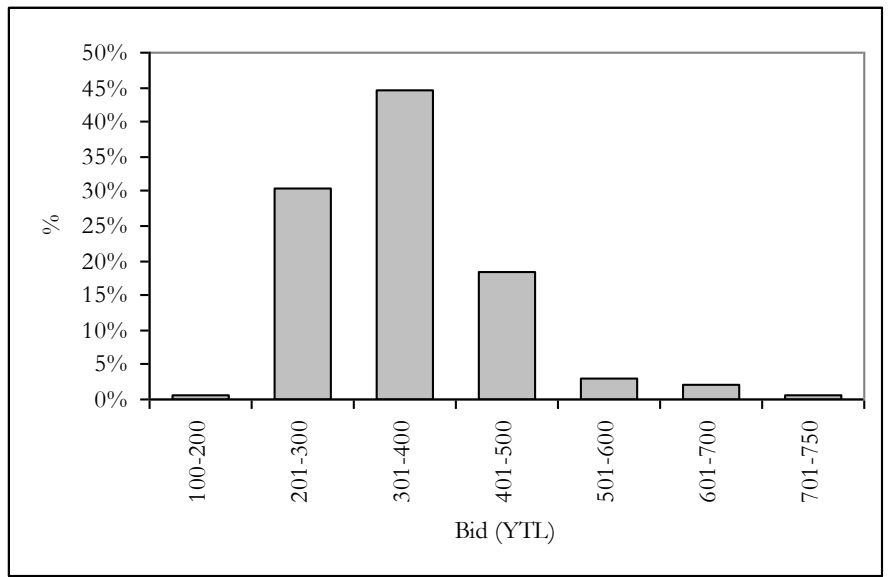

Figure 5. Distribution of the bids (in Turkish Lira) given to the orders from Eskişehir to Adana at the ESO Logistics Center during December 2003-March 2004

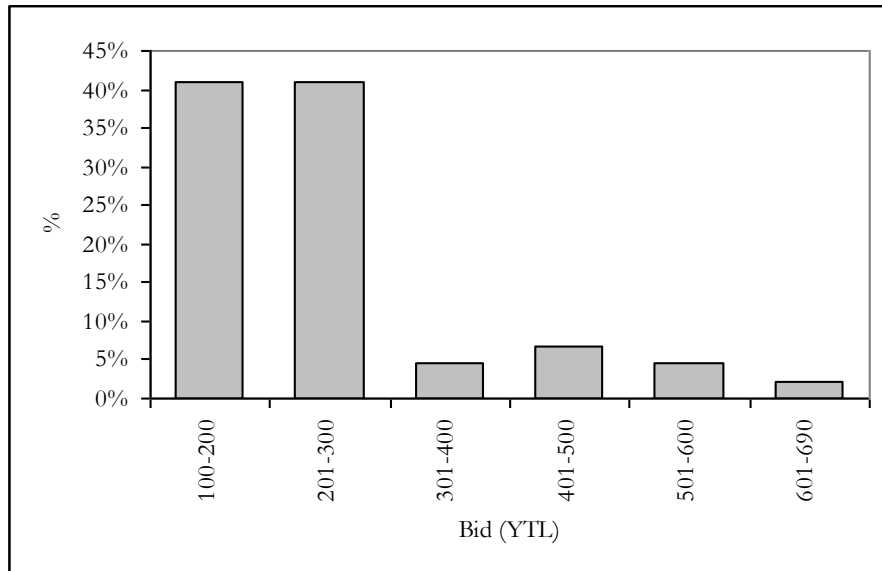

Figure 6. Distribution of the prices (in Turkish Lira) paid to the orders from Eskişehir to Adana at the ESO Logistics Center during December 2003-March 2004

The number of carriers engaging in an auction is concealed and all the carriers are assumed to have the same belief about the probability distribution of the number of bidders joining an auction and the probability that each bidder is a local or an in-transit carrier. Each bidder makes his bid according to this common belief. Since the probability distribution of the number of bidders joining an auction and the probability of the type of each bidder are common beliefs, each bidder uses the same probability distribution to determine his bid. This distribution is based on the distributions of the costs of carriers, and also the number and the type of carriers joining an auction. Therefore, this is a symmetric auction although there are two types of bidders.

An auction is opened at the instant when an order arrives and there are carriers waiting to bid. Since the probability that two orders arrive exactly at the same time is very small, only one auction is opened at a time. The auction duration is very short compared to the time scale of order and carrier arrivals and therefore it is taken to be instantaneous. 
If there are no carriers at the LC, the order is registered by the system and stays there until the order is cancelled by the shipper or given to the carrier that arrives first at the designated market price which is assumed to be equal to $c_{1}^{\prime}$. Note that a shipper can abandon the LC if it does not want to pay the market price. In the ESO case, the market price is listed for various destinations by the carriers' cooperation and shown in Figure 2.

In this setting, the assumptions of the auction and carriers guarantee that the optimal strategy for a carrier is to bid her actual cost (Vickrey, 1961) to maximize her expected gain. For an extensive review of auction theory, the reader is referred to (Klemperer, 1999). Pitfalls for bidders, equivalences among auction institutions and comparison of auctions are summarized in (Milgrom, 1989).

\section{Analysis of the Model}

We analyze the system in two stages. First we analyze the auction part based on the given number of carriers that bid for an order. Since the carriers bid their actual costs to maximize their expected gain in the Vickrey auction, we determine the expected auction price and the expected profit of the carrier that receives the order by using order statistics. In the second part of the analysis, we model the random order and carrier arrival-departure processes and determine the steady-state probability distribution of the number of carriers in the logistics center. Finally, by combining the results of these two parts, we determine various metrics that measure the average performance of the system in the long run.

\subsection{Analysis of an Auction}

Expected Price. Let $p\left(n_{0}, n_{1}, n_{2}\right)$ denote the expected price when $n_{0}$ orders, $n_{1}$ type 1 , and $n_{2}$ type 2 carriers are at the logistics center just before an order is matched with a carrier. Note that when there are no carriers available at the logistics center, a waiting order is matched with an arriving carrier at the market price, i.e.,

$$
p\left(n_{0}, 0,0\right)=c_{1}^{\prime}, n_{0} \geq 1
$$

Similarly, when there is only one carrier available, an arriving order is priced at $c_{1}^{\prime}$. That is,

$$
p(0,1,0)=p(0,0,1)=c_{1}^{\prime} .
$$

When there are $N_{1}(t)+N_{2}(t)=n_{1}+n_{2}, n_{1}+n_{2} \geq 2$ carriers at time $t$, an auction is conducted as soon as a new order arrives. Let $J_{(1)} \leq J_{(2)} \leq \ldots \leq J_{\left(n_{1}+n_{2}\right)}$ be the order statistics defined on the actual costs of $n_{1}+n_{2}$ carriers. Since carriers bid their actual costs and they are paid at the second lowest bid, the expected auction price is the expected value of the second minimum of the bidders' costs:

$$
p\left(0, n_{1}, n_{2}\right)=E\left[J_{(2)} \mid N_{1}(t)=n_{1}, N_{2}(t)=n_{2}\right], n_{1}+n_{2} \geq 2 .
$$

Since the distributions of the costs of type 1 and type 2 carriers are different, we consider different cases to determine the expectations of order statistics based on the cost distributions. Proposition 1 gives the expected auction price following standard results in order statistics. 
Proposition 1: The expected price when there are $n_{0}$ orders, $n_{1}$ type 1 , and $n_{2}$ type 2 carriers at the logistics center, $p\left(n_{0}, n_{1}, n_{2}\right)$, is:

$$
p\left(n_{0}, n_{1}, n_{2}\right)=\left\{\begin{array}{ll}
c_{2}+\int_{c_{2}}^{c_{2}^{\prime}}\left[1-F_{2}(x)\right]^{n_{2}-1} n_{2} F_{2}(x) d x+\int_{c_{2}}^{c_{2}^{\prime}}\left[1-F_{2}(x)\right]^{n_{2}} d x & n_{0}=0, n_{2}>1 \\
c_{1}+\int_{c_{1}}^{c_{1}^{\prime}}\left[1-F_{1}(x)\right]^{n_{1}} d x & n_{0}=0, n_{1}>0, n_{2}=1 \\
c_{1}+\int_{c_{1}}^{c_{1}^{\prime}}\left[1-F_{1}(x)\right]^{n_{1}-1} n_{1} F_{1}(x) d x+\int_{c_{1}}^{c_{1}^{\prime}}\left[1-F_{1}(x)\right]^{n_{1}} d x & n_{0}=0, n_{1}>1, n_{2}=0 \\
c_{1}^{\prime} & n_{0}=0, n_{1}=0, n_{2}=1 \\
& n_{0}=0, n_{1}=1, n_{2}=0 \\
& n_{0} \geq 1, n_{1}=0, n_{2}=0
\end{array} .\right.
$$

We next show how the expected price changes when the number of carriers and orders change in a given auction.

Lemma 1: The expected price, $p\left(n_{0}, n_{1}, n_{2}\right)$, is non-increasing in $n_{1}$ and $n_{2}$.

Proof. The proof of Lemma 1 is given in the Appendix.

Figure 7 shows the realized average auction price according to the number of bidders joining the auction for orders from Eskişehir to Adana at the ESO center. As the figure depicts, the realized average auction price at ESO decreases with the increasing number of bids as Lemma 1 predicts.

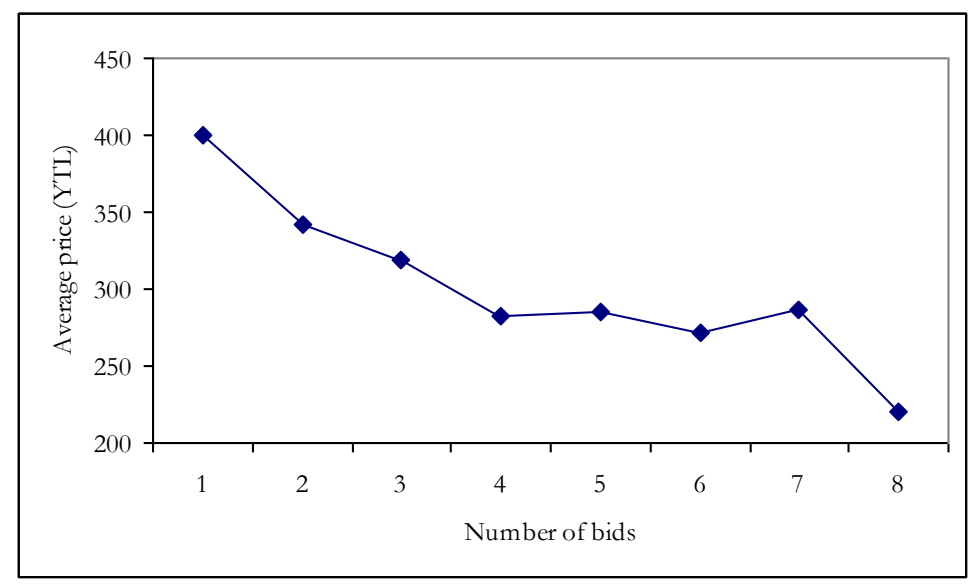

Figure 7. Number of bids and the average auction prices for orders from Eskişehir to Adana

Expected Profit of the Winner. The profit of the winner is the difference between the price and her actual cost. The expected profit when there are $n_{0}$ orders, $n_{1}$ type 1 , and $n_{2}$ type 2 carriers in the LC is denoted with $q\left(n_{0}, n_{1}, n_{2}\right)$.

When there are waiting orders and no carriers, the profit depends on the type of the winning carrier. If the winner is of Type $i$, then $q\left(n_{0}, 0,0\right)=c_{1}^{\prime}-\bar{c}_{i}, n_{0} \geq 1$. Similarly, $q(0,1,0)=c_{1}^{\prime}-\overline{c_{1}}$ and $q(0,0,1)=c_{1}^{\prime}-\overline{c_{2}}$.

If there are $n_{1}+n_{2} \geq 2$ carriers, the winning carrier's cost is the lowest among all the bidders. Therefore,

$$
q\left(0, n_{1}, n_{2}\right)=p\left(0, n_{1}, n_{2}\right)-E\left[J_{(1)} \mid N_{1}(t)=n_{1}, N_{2}(t)=n_{2}\right] .
$$


If there are no orders or carriers available, there will be no auction and we set $p(0,0,0)=q(0,0,0)=0$.

Based on the distributions of carriers' costs, Proposition 2 gives the expected profit of the winner.

Proposition 2: The expected profit of the winner, $q\left(n_{0}, n_{1}, n_{2}\right)$, when there are there are $n_{0}$ orders, $n_{1}$ type 1 , and $n_{2}$ type 2 carriers in the LC is:

$$
q\left(n_{0}, n_{1}, n_{2}\right)= \begin{cases}\int_{c_{2}}^{c_{2}}\left[1-F_{2}(x)\right]^{n_{2}-1} n_{2} F_{2}(x) d x & n_{0}=0, n_{2}>1 \\ c_{1}^{\prime}+\int_{c_{1}}^{c_{1}^{\prime}}\left[1-F_{1}(x)\right]^{n_{1}} d x-\overline{c_{2}} & n_{0}=0, n_{1}>0, n_{2}=1 \\ c_{1}^{\prime} & n_{0}=0, n_{1}>1, n_{2}=0 \\ \int_{c_{1}}\left[1-F_{1}(x)\right]^{n_{1}-1} n_{1} F_{1}(x) d x & \left(n_{0}=0, n_{1}=1, n_{2}=0\right) \text { or }\left(n_{0} \geq 1, n_{1}=0, n_{2}=0 \text { and Type } 1 \text { arrives }\right) \\ c_{1}^{\prime}-\overline{c_{1}} & \left(n_{0}=0, n_{1}=0, n_{2}=1\right) \text { or }\left(n_{0} \geq 1, n_{1}=0, n_{2}=0 \text { and Type } 2 \text { arrives }\right) \\ c_{1}^{\prime}-\overline{c_{2}} & \end{cases}
$$

\subsection{Analysis of the Order-Carrier Arrival-Departure Processes}

In the preceding analysis, we determined the auction price and the profit depending on the number of carriers of each type and the number of orders. Since the number of carriers and orders change dynamically with random arrivals and abandonment of orders and carriers, we next analyze the dynamics of the system to determine the steady-state distribution of the number of carriers of each type and the number of orders at the logistics center.

Let the state of the system at time $t$ be $S(t)=\left(N_{0}(t), N_{1}(t), N_{2}(t)\right)$. Since the interarrival and abandonment times of carriers and orders are exponential random variables, the process $\{S(t), t \geq 0\}$ is a continuous-time Markov Chain. The process is ergodic and a stationary distribution exists. The steadystate probabilities are defined as

$$
\pi\left(n_{0}, n_{1}, n_{2}\right)=\lim _{t \rightarrow \infty} \operatorname{Prob}\left\{N_{0}(t)=n_{0}, N_{1}(t)=n_{1}, N_{2}(t)=n_{2}\right\} .
$$

All the relevant steady-state performance measures are determined based on the steady-state probabilities. Since an auction is conducted instantaneously, it is not possible to observe $N_{0}(t)+N_{1}(t) \geq 1$ when $N_{0}(t) \geq 1$. As a result, $\pi\left(n_{0}, n_{1}, n_{2}\right)=0$ when $n_{0} \geq 1$ and $n_{1}+n_{2} \geq 1$.

In order to analyze the performance of this system numerically, the state-transition equations are derived and the steady-state probabilities are calculated by truncating the state space at state $\left(K_{0}, K_{1}, K_{2}\right)$. $K_{1}$ and $K_{2}$ can also be considered as the maximum number of type 1 and type 2 carriers the LC can accommodate and $K_{0}$ can be considered as the maximum number of orders that can be accepted by the system. There are a total of $K_{0}+\left(K_{1}+1\right)\left(K_{2}+1\right)$ states in the resulting state space $S(t)=\left(N_{0}(t), N_{1}(t), N_{2}(t)\right)$.

\section{State-Transition Equations}

The state transition diagram of the logistics center is shown in Figure 8 for a specific system with $K_{0}=K_{1}=K_{2}=5$. Since we assume $c_{1}>c_{2}^{\prime}$, the transition from one state to another when an order comes, 
depends on the number of type 2 carriers. In addition, some of the orders and carriers are rejected due to the capacity constraints. Accordingly, we analyze the internal and boundary transitions separately depending on the number of carriers of each type.

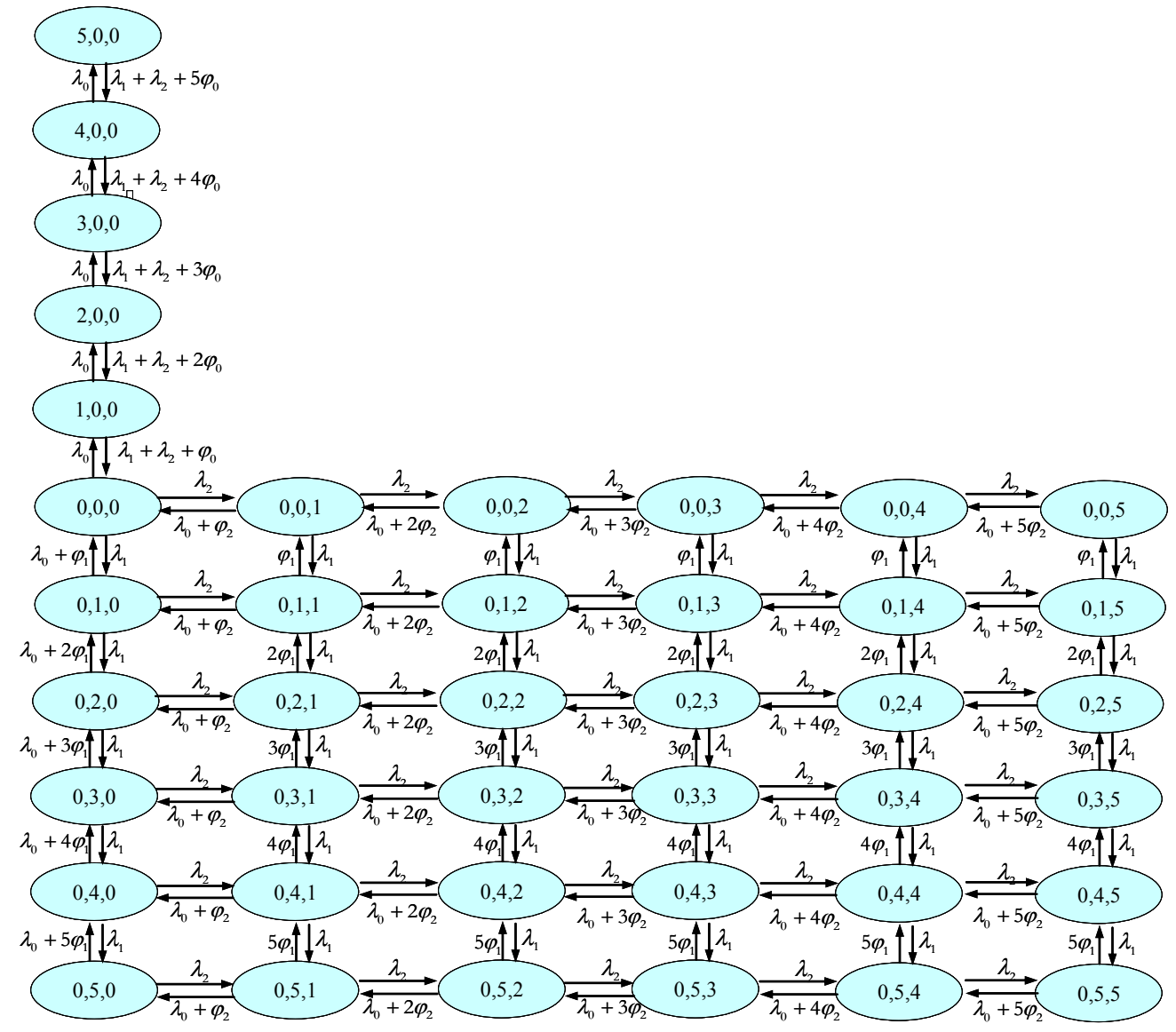

Figure 8. State transition diagram of a logistics spot market with $K_{0}=K_{1}=K_{2}=5$.

When the LC is empty, the state changes only with the arrivals of carriers or orders. That is,

$$
\left(\lambda_{0}+\lambda_{1}+\lambda_{2}\right) \pi(0,0,0)=\left(\lambda_{0}+\varphi_{2}\right) \pi(0,0,1)+\left(\lambda_{0}+\varphi_{1}\right) \pi(0,1,0)+\left(\lambda_{1}+\lambda_{2}+\varphi_{0}\right) \pi(1,0,0) .
$$

When there are no carriers but waiting orders and the order capacity is still available to accept new orders, the states change with an arrival of carriers, arrival of orders, or with possible abandonment of waiting orders:

$$
\begin{array}{r}
\left(\lambda_{0}+\lambda_{1}+\lambda_{2}+n_{0} \varphi_{0}\right) \pi\left(n_{0}, 0,0\right)=\left(\lambda_{1}+\lambda_{2}+\left(n_{0}+1\right) \varphi_{0}\right) \pi\left(n_{0}+1,0,0\right)+\lambda_{0} \pi\left(n_{0}-1,0,0\right), \\
n_{0}=1, \ldots, K_{0}-1 .
\end{array}
$$

If there are no carriers and the capacity limit of orders is reached so that no additional order can be accepted to the logistics center,

$$
\left(\lambda_{1}+\lambda_{2}+K_{0} \varphi_{0}\right) \pi\left(K_{0}, 0,0\right)=\lambda_{0} \pi\left(K_{0}-1,0,0\right) .
$$

When there is at least one type 2 carrier and there are no type 1 carriers at LC, the order is taken by one of type 2 carriers: 


$$
\begin{gathered}
\left(\lambda_{0}+\lambda_{1}+\lambda_{2}+n_{2} \varphi_{2}\right) \pi\left(0,0, n_{2}\right)=\lambda_{2} \pi\left(0,0, n_{2}-1\right)+\left(\lambda_{0}+\left(n_{2}+1\right) \varphi_{2}\right) \pi\left(0,0, n_{2}+1\right)+\varphi_{1} \pi\left(0,1, n_{2}\right) \\
n_{2}=1, \ldots, K_{2}-1
\end{gathered}
$$

When the system capacity for type 2 carriers is reached and there are no type 1 carriers:

$$
\left(\lambda_{0}+\lambda_{1}+K_{2} \varphi_{2}\right) \pi\left(0,0, K_{2}\right)=\lambda_{2} \pi\left(0,0, K_{2}-1\right)+\varphi_{1} \pi\left(0,1, K_{2}\right) .
$$

Similarly, when there is at least one type 1 carrier and there are no type 2 carriers at LC, the order is taken by one of the type 1 carriers:

$$
\begin{aligned}
& \left(\lambda_{0}+\lambda_{1}+\lambda_{2}+n_{1} \varphi_{1}\right) \pi\left(0, n_{1}, 0\right)=\lambda_{1} \pi\left(0, n_{1}-1,0\right)+\left(\lambda_{0}+\varphi_{2}\right) \pi\left(0, n_{1}, 1\right)+\left(\lambda_{0}+\left(n_{1}+1\right) \varphi_{1}\right) \pi\left(0, n_{1}+1,0\right), \\
& n_{1}=1, \ldots, K_{1}-1 \text {. }
\end{aligned}
$$

In this setting, when the system capacity for type 1 carriers is reached and there are no type 2 carriers:

$$
\left(\lambda_{0}+\lambda_{2}+K_{1} \varphi_{1}\right) \pi\left(0, K_{1}, 0\right)=\lambda_{1} \pi\left(0, K_{1}-1,0\right)+\left(\lambda_{0}+\varphi_{2}\right) \pi\left(0, K_{1}, 1\right) .
$$

When at least one carrier of each type is at the logistics center and the capacity limits are not reached, the state changes with the arrival of carriers, orders and also with the abandonment of carriers:

$$
\begin{aligned}
& \left(\lambda_{0}+\lambda_{1}+\lambda_{2}+n_{1} \varphi_{1}+n_{2} \varphi_{2}\right) \pi\left(0, n_{1}, n_{2}\right)= \\
& \lambda_{1} \pi\left(0, n_{1}-1, n_{2}\right)+\lambda_{2} \pi\left(0, n_{1}, n_{2}-1\right)+\left(\lambda_{0}+\left(n_{2}+1\right) \varphi_{2}\right) \pi\left(0, n_{1}, n_{2}+1\right)+\left(n_{1}+1\right) \varphi_{1} \pi\left(0, n_{1}+1, n_{2}\right), \\
& n_{1}=1, \ldots, K_{1}-1 ; n_{2}=1, \ldots, K_{2}-1 .
\end{aligned}
$$

When the capacity for type 1 carriers is full and there is at least one type 2 carrier, the state changes with the arrival of type 2 carriers and orders, and possible abandonment of both type 1 and type 2 carriers. Then,

$$
\begin{array}{r}
\left(\lambda_{0}+\lambda_{2}+K_{1} \varphi_{1}+n_{2} \varphi_{2}\right) \pi\left(0, K_{1}, n_{2}\right)=\lambda_{1} \pi\left(0, K_{1}-1, n_{2}\right)+\lambda_{2} \pi\left(0, K_{1}, n_{2}-1\right)+\left(\lambda_{0}+\left(n_{2}+1\right) \varphi_{2}\right) \pi\left(0, K_{1}, n_{2}+1\right), \\
n_{2}=1, \ldots, K_{2}-1 .
\end{array}
$$

Similarly, when there is no available space for type 2 carriers and only type 1 carriers can be accepted,

$$
\begin{gathered}
\left(\lambda_{0}+\lambda_{1}+n_{1} \varphi_{1}+K_{2} \varphi_{2}\right) \pi\left(0, n_{1}, K_{2}\right)=\lambda_{1} \pi\left(0, n_{1}-1, K_{2}\right)+\lambda_{2} \pi\left(0, n_{1}, K_{2}-1\right)+\left(n_{1}+1\right) \varphi_{1} \pi\left(n_{1}+1, K_{2}, 0\right) \\
n_{1}=1, \ldots, K_{1}-1 .
\end{gathered}
$$

Finally, when the capacity limits of carriers of both types are reached, then

$$
\left(\lambda_{0}+K_{1} \varphi_{1}+K_{2} \varphi_{2}\right) \pi\left(0, K_{1}, K_{2}\right)=\lambda_{1} \pi\left(0, K_{1}-1, K_{2}\right)+\lambda_{2} \pi\left(0, K_{1}, K_{2}-1\right) .
$$

The solution of the state transition equations (6)-(16) with the normalization condition

$$
\sum_{n_{0}=0}^{K_{0}} \sum_{n_{1}=0}^{K_{1}} \sum_{n_{2}=0}^{K_{2}} \pi\left(n_{0}, n_{1}, n_{2}\right)=1
$$

yields the steady-state probabilities. 


\section{Performance Measures}

Once the steady-state probabilities are determined, a number of performance measures related to the carriers, shippers, and the Logistics Center can be determined. In order to determine the effects of the logistics center, we use the average expected price given for an order and the average expected auction price when at least two carriers compete to get an order as the main performance measures.

In order to study the effects of the logistics center on the carriers, we determine the average profit of a carrier that receives an order, the fraction of type $i$ carriers that are awarded with an order, and the proportion of the number of type $i$ carriers in the total number of carriers awarded with an order. The performance of the logistics center is also evaluated by determining the average number of carriers and orders.

Average Expected Price. Let $\bar{P}$ be the average expected price paid for an order. Since $p\left(n_{0}, n_{1}, n_{2}\right)$ is calculated by conditioning on the number of carriers and orders, $\bar{P}$ is determined by using the steady state probability distribution of the number of carriers and the number of orders present at the logistics center just before an order is matched with a carrier. Note that an auction is conducted if there are at least two carriers available at the logistics center. Otherwise, the market price is paid. Let $\eta_{0}, \eta_{1}$, and $\eta_{2}$ denote the number of orders, type 1 and type 2 carriers available at the logistics center when an order is matched with a carrier in the steady state.

$$
\bar{P}=\sum_{n_{0}=0}^{K_{0}} \sum_{n_{1}=0}^{K_{1}} \sum_{n_{2}=0}^{K_{2}} p\left(n_{0}, n_{1}, n_{2}\right) \operatorname{Prob}\left[\eta_{0}=n_{0}, \eta_{1}=n_{1}, \eta_{2}=n_{2}\right]
$$

The steady-state distribution of $\eta_{0}, \eta_{1}$, and $\eta_{2}$ can be determined from the steady-state distribution of $N_{0}$, $N_{1}$, and $N_{2}$ as

$$
\begin{aligned}
& \operatorname{Prob}\left[\eta_{0}=0, \eta_{1}=n_{1}, \eta_{2}=n_{2}\right]=\frac{\lambda_{0}}{\lambda_{A}} \pi\left(0, n_{1}, n_{2}\right) \text { and } \\
& \operatorname{Prob}\left[\eta_{0}=n_{0}, \eta_{1}=0, \eta_{2}=0\right]=\frac{\left(\lambda_{1}+\lambda_{2}\right)}{\lambda_{A}} \pi\left(n_{0}, 0,0\right)
\end{aligned}
$$

where $\lambda_{A}$ is the expected number of carriers that are matched with an order per unit time in the long run and given in Equation (24). Then $\bar{P}$ is evaluated as

$$
\bar{P}=\frac{\left(\lambda_{1}+\lambda_{2}\right)}{\lambda_{A}} \sum_{n_{0}=1}^{K_{0}} c_{1}^{\prime} \pi\left(n_{0}, 0,0\right)+\frac{\lambda_{0}}{\lambda_{A}} \sum_{n_{1}=0}^{K_{1}} \sum_{n_{2}=0}^{K_{2}} p\left(0, n_{1}, n_{2}\right) \pi\left(0, n_{1}, n_{2}\right) .
$$

Average Expected Auction Price. Let $\bar{P}_{A}$ be the average expected auction price when there are at least two carriers at the logistics center. Therefore, the average expected auction price is 


$$
\bar{P}_{A}=\sum_{n_{1}+n_{2} \geq 2} p\left(0, n_{1}, n_{2}\right) \operatorname{Prob}\left[\eta_{1}=n_{1}, \eta_{2}=n_{2} \mid \eta_{1}+\eta_{2} \geq 2\right]=\frac{\sum_{n_{1}+n_{2} \geq 2} p\left(0, n_{1}, n_{2}\right) \pi\left(0, n_{1}, n_{2}\right)}{\sum_{n_{1}+n_{2} \geq 2} \pi\left(0, n_{1}, n_{2}\right)} .
$$

The Proportion of Carriers that Take an Order. Not all the arriving carriers are awarded with an order. In our model, the arriving carriers can abandon the system after waiting some time and a few of them are rejected due to the capacity constraint. All the carriers that are accepted by the system and wait without abandoning the system are awarded with an order. Let $f_{i}$ be the proportion of type $i$ carriers that take an order to the total number of arriving carriers of the same type for $i=1,2$. Then $f_{i}$ can be calculated as the ratio of the order arrivals that are not rejected due to the capacity constraint and wait without abandoning the system in the total arrivals:

$$
\begin{aligned}
& f_{1}=1-\sum_{n_{2}=0}^{K_{2}} \pi\left(0, K_{1}, n_{2}\right)-\sum_{n_{1}=0}^{K_{1}} \sum_{n_{2}=0}^{K_{2}} n_{1} \frac{\varphi_{1}}{\lambda_{1}} \pi\left(0, n_{1}, n_{2}\right), \\
& f_{2}=1-\sum_{n_{1}=0}^{K_{1}} \pi\left(0, n_{1}, K_{2}\right)-\sum_{n_{1}=0}^{K_{1}} \sum_{n_{2}=0}^{K_{2}} n_{2} \frac{\varphi_{2}}{\lambda_{2}} \pi\left(0, n_{1}, n_{2}\right)
\end{aligned}
$$

Then the expected number of carriers that are matched with an order per unit time in the long run can be written in term of $f_{1}$ and $f_{2}$ as

$$
\lambda_{A}=\lambda_{1} f_{1}+\lambda_{2} f_{2}
$$

As a result, the fraction of carriers that are awarded with an order in the total number of arriving carriers is $\frac{\lambda_{1} f_{1}+\lambda_{2} f_{2}}{\lambda_{1}+\lambda_{2}}$. Similarly, since the total order arrival rate is $\lambda_{0}, \lambda_{A} / \lambda_{0}$ of the arriving orders are matched with a carrier, while the remaining $1-\lambda_{A} / \lambda_{0}$ of them either abandon the system or are rejected due to the order capacity constraint of the logistics center.

Distribution of Orders between Type 1 and Type 2 Carriers. Let $d_{i}$ be the proportion of the number of type $i$ carriers that are awarded with an order to the total number of carriers awarded with an order. Then

$$
d_{i}=\frac{\lambda_{i} f_{i}}{\lambda_{1} f_{1}+\lambda_{2} f_{2}}, i=1,2
$$

Average Expected Profit of Carriers. Let us define $\bar{Q}_{i}$ as the average expected profit of a type $i$ carrier that is awarded with an order $i=1,2$. Note that if there is at least one type 2 bidder, then the winner will be of type 2 . Equivalently, the winner is of type 1 only when there are no type 2 bidders. Then, 


$$
\begin{gathered}
\bar{Q}_{1}=\frac{\lambda_{0} \sum_{n_{1}=1}^{K_{1}} q\left(0, n_{1}, 0\right) \pi\left(0, n_{1}, 0\right)+\lambda_{1} \sum_{n_{0}=1}^{K_{0}}\left(c_{1}^{\prime}-\overline{c_{1}}\right) \pi\left(n_{0}, 0,0\right)}{\lambda_{0} \sum_{n_{1}=1}^{K_{1}} \pi\left(0, n_{1}, 0\right)+\lambda_{1} \sum_{n_{0}=1}^{K_{0}} \pi\left(n_{0}, 0,0\right)} \text { and } \\
\bar{Q}_{2}=\frac{\lambda_{0} \sum_{n_{1}=0}^{K_{1}} \sum_{n_{2}=1}^{K_{2}} q\left(0, n_{1}, n_{2}\right) \pi\left(0, n_{1}, n_{2}\right)+\lambda_{2} \sum_{n_{0}=1}^{K_{0}}\left(c_{1}^{\prime}-\overline{c_{2}}\right) \pi\left(n_{0}, 0,0\right)}{\lambda_{0} \sum_{n_{1}=0}^{K_{1}} \sum_{n_{2}=1}^{K_{2}} \pi\left(0, n_{1}, n_{2}\right)+\lambda_{2} \sum_{n_{0}=1}^{K_{0}} \pi\left(n_{0}, 0,0\right)} .
\end{gathered}
$$

Let $\bar{Q}$ be the average expected profit of a carrier (type 1 or type 2 ) that is awarded with an order. Since $\left(1-f_{i}\right)$ of all the arriving carriers leave the system with a profit of zero and $f_{i}$ of them make a profit of $\bar{Q}_{i}$ on the average, for an arriving carrier, the average profit is $f_{i} \bar{Q}_{i}, i=1,2$.. Accordingly, the average profit for a carrier that is awarded with an order is

$$
\bar{Q}=\frac{\lambda_{1} f_{1} \bar{Q}_{1}+\lambda_{2} f_{2} \bar{Q}_{2}}{\lambda_{1} f_{1}+\lambda_{2} f_{2}}
$$

Average Number of Carriers and Orders. The average number of orders and carriers of type $i$ available at the logistics center in steady state are denoted by $\bar{N}_{0}$ and $\bar{N}_{i}, i=1,2$. The steady state distribution of the number of carriers and orders yield the average number of carriers and orders directly:

$$
\bar{N}_{0}=\sum_{n_{0}=1}^{K_{0}} n_{0} \pi\left(n_{0}, 0,0\right) \text { and } \bar{N}_{i}=\sum_{n_{1}=0}^{K_{1}} \sum_{n_{2}=0}^{K_{2}} n_{i} \pi\left(0, n_{1}, n_{2}\right), i=1,2
$$

\section{Performance Evaluation}

In this section we evaluate the performance of the logistics market numerically and analytically. We first summarize the results of our numerical experiments and then present our analytical results regarding the effects of system parameters on the average expected auction price.

\subsection{Numerical Results}

Setting. In our numerical experiments, we consider a specific logistics market where the cost distributions of local and in-transit carriers are uniform. Accordingly,

$$
F_{i}(x)=\frac{x-c_{i}}{c_{i}^{\prime}-c_{i}}, \quad c_{i} \leq x \leq c_{i}^{\prime}, i=1,2
$$

Note that it is also possible to handle other distributions since Equations (2) and (4) can be evaluated for given cost distributions. For uniformly distributed costs, $p\left(n_{0}, n_{1}, n_{2}\right)$ and $q\left(n_{0}, n_{1}, n_{2}\right)$ can be written in closed form as 


$$
p\left(n_{0}, n_{1}, n_{2}\right)=\left\{\begin{array}{ll}
c_{2}+\frac{2\left(c_{2}^{\prime}-c_{2}\right)}{n_{2}+1} & n_{0}=0, n_{2}>1 \\
c_{1}+\frac{\left(c_{1}^{\prime}-c_{1}\right)}{n_{1}+1} & n_{0}=0, n_{1}>0, n_{2}=1 \\
c_{1}+\frac{2\left(c_{1}^{\prime}-c_{1}\right)}{n_{1}+1} & n_{0}=0, n_{1}>1, n_{2}=0 \\
& n_{0}=0, n_{1}=0, n_{2}=1 \\
n_{0}=0, n_{1}=1, n_{2}=0 \\
n_{0} \geq 1, n_{1}=0, n_{2}=0
\end{array} \quad q\left(n_{0}, n_{1}, n_{2}\right)=\left\{\begin{array}{ll}
\frac{c_{2}^{\prime}-c_{2}}{n_{2}+1} & n_{0}=0, n_{2}>1 \\
c_{1}+\frac{\left(c_{1}^{\prime}-c_{1}\right)}{n_{1}+1}-\overline{c_{2}} & n_{0}=0, n_{1}>0, n_{2}=1 \\
\frac{c_{1}^{\prime}-c_{1}}{n_{1}+1} & n_{0}=0, n_{1}>1, n_{2}=0 \\
c_{1}^{\prime}-\overline{c_{1}} & n_{0}=0, n_{1}=1, n_{2}=0 \\
c_{1}^{\prime}-\overline{c_{2}} & n_{0}=0, n_{1}=0, n_{2}=1
\end{array} .\right.\right.
$$

Parameterization. In order to analyze the system for different parameter sets, we first set the average cost and the arrival rate of local carriers to one without loss of generality, i.e., $\overline{c_{1}}=1$ and $\lambda_{1}=1$. Furthermore, we assume that the cost distribution is defined in the range $\overline{c_{1}}(1 \pm \Delta)$. That is, $c_{1}=1-\Delta$ and $c_{1}^{\prime}=1+\Delta$. We set the average cost of the in-transit carriers as $\overline{c_{2}}=(1-k) \overline{c_{1}}$ and $c_{2}=\overline{c_{2}}(1-\Delta)$ and $c_{2}^{\prime}=\bar{c}_{2}(1+\Delta)$. In this parameterization, the cost distributions are not overlapping if $\frac{\Delta}{\Delta+1} \leq \frac{k}{2}$. Finally, since in-transit carriers are more likely to abandon earlier than the local carriers, we set $\varphi_{1}=2 \varphi_{2}$. Then we evaluate the performance of the system for different values of $\lambda_{1}, \lambda_{2}, \varphi_{0}, \varphi_{1}, k$, and $\Delta$. Table 1 reports the parameter values used in the numerical experiments.

\begin{tabular}{|c|c||c|c|}
\hline Parameter & Values & Parameter & Values \\
\hline$\lambda_{0}$ & $\{0.25,0.5,1,1.5,2\}$ & $k$ & $\{0.5,0.6,0.7,0.8\}$ \\
\hline$\lambda_{1}$ & 1 & $\Delta$ & $\{0.1,0.2,0.3\}$ \\
\hline$\lambda_{2}$ & $\{0,0.5,1,1.5,2\}$ & $c_{1}$ & $1-\Delta$. \\
\hline$\varphi_{0}$ & $\{0.2,0.4,0.6,0.8,1\}$ & $c_{1}^{\prime}$ & $1+\Delta$. \\
\hline$\varphi_{1}$ & $\{0.2,0.4,0.6,0.8,1\}$ & $c_{2}$ & $(1-k)(1-\Delta)$. \\
\hline$\varphi_{2}$ & $1 / 2 \varphi_{1}$ & $c_{2}^{\prime}$ & $(1-k)(1+\Delta)$. \\
\hline
\end{tabular}

Table 1. Parameter values for numerical experiments

The capacities for orders and carriers, $K_{0}, K_{1}$, and $K_{2}$, are set to 30 . For the above parameter values, the rejection probabilities for orders and carriers are less than $10^{-13}$ and therefore the results are not affected by truncating the state space.

With this parameter set, we evaluate the performance of the logistics market for 7500 different cases. Cases with $\lambda_{2}=0$ correspond to a logistics market with only local carriers and therefore they allow us to evaluate the effect of allowing in-transit carriers on local carriers and firms.

We summarize our findings below. In all of the tables, two parameters are changed at a time. Then the average values of performance measures in all the cases with the specific values of these parameters are reported. We focus on the effects of the arrival rates of local and in-transit carriers and also the difference between the average costs and the variability around these averages.

Effect on Firms. Without a logistics spot market, a firm pays the market price to a carrier. The logistics center is expected to lower the price paid by the firms. Table 2 shows the percentage reduction in 
the price paid by the firms with respect to the market price $P_{M}=c_{1}^{\prime}$ for different type 2 carrier arrival rates, order arrival rates, abandonment rates, and cost distributions.

\begin{tabular}{|r|rrrrr|}
\hline$\frac{P_{M}-\bar{P}}{P_{M}}$ & \multicolumn{5}{|c|}{$\lambda_{0}$} \\
\hline$\lambda_{2}$ & 0.25 & 0.50 & 1.00 & 1.50 & 2.00 \\
\hline 0.00 & $8 \%$ & $6 \%$ & $3 \%$ & $2 \%$ & $1 \%$ \\
0.50 & $15 \%$ & $13 \%$ & $8 \%$ & $5 \%$ & $3 \%$ \\
1.00 & $25 \%$ & $22 \%$ & $16 \%$ & $11 \%$ & $7 \%$ \\
1.50 & $34 \%$ & $31 \%$ & $24 \%$ & $18 \%$ & $13 \%$ \\
2.00 & $42 \%$ & $38 \%$ & $32 \%$ & $26 \%$ & $20 \%$ \\
\hline
\end{tabular}

\begin{tabular}{|r|rrrrr|}
\hline$\frac{P_{M}-\bar{P}}{P_{M}}$ & \multicolumn{5}{|c|}{$\varphi_{2}$} \\
\hline$\varphi_{0}$ & 0.40 & 0.80 & 1.20 & 1.60 & 2.00 \\
\hline 0.20 & $30 \%$ & $19 \%$ & $13 \%$ & $10 \%$ & $7 \%$ \\
0.40 & $31 \%$ & $20 \%$ & $14 \%$ & $10 \%$ & $8 \%$ \\
0.60 & $31 \%$ & $20 \%$ & $14 \%$ & $11 \%$ & $8 \%$ \\
0.80 & $32 \%$ & $21 \%$ & $15 \%$ & $11 \%$ & $8 \%$ \\
1.00 & $32 \%$ & $21 \%$ & $15 \%$ & $11 \%$ & $9 \%$ \\
\hline
\end{tabular}

\begin{tabular}{|r|rrrr|}
\hline$\frac{P_{M}-\bar{P}}{P_{M}}$ & \multicolumn{4}{|c|}{$k$} \\
\hline$\Delta$ & 0.5 & 0.6 & 0.7 & 0.8 \\
\hline 0.1 & $12 \%$ & $13 \%$ & $15 \%$ & $16 \%$ \\
0.2 & $15 \%$ & $16 \%$ & $18 \%$ & $19 \%$ \\
0.3 & $17 \%$ & $19 \%$ & $20 \%$ & $21 \%$ \\
\hline
\end{tabular}

Table 2. Percentage Reduction in the Average Expected Price

Table 2 indicates that the benefit of the logistics spot market on lowering the price paid by the firms will be limited when in-transit carriers are not utilized. In this case, the percentage reduction will be between $8 \%$ and $1 \%$ depending on the order arrival rate. As the order arrival rate increases, the number of carriers participating in the auctions decreases and as a result the price paid by the carriers will not decrease substantially. When in-transit carriers are allowed, the prices decrease quickly as their arrival rate increases. Since waiting orders are matched with an arriving carrier at the market price, the order abandonment rate does not affect the expected average price. However, keeping the in-transit carriers longer at the logistics market by decreasing their abandonment rate decreases the price substantially.

The difference between the average costs of local and in-transit carriers and the variability of the cost distributions have limited effect on the prices. This is due to using a second price auction. As long as the second minimum bid is submitted by a local carrier, the difference in average costs will not have any effect on the price paid by a carrier. Similarly, the variability around the mean will be effective only when the auction is conducted among the same type carriers.

Effect on Local Carriers. Table 2 shows that allowing in-transit carriers will be effective in lowering the prices paid by the firms. However, the local carriers will be affected negatively since they will lose some of the orders to in-transit carriers. Table 3 quantifies the percentage of local carriers receiving an order and the proportion of local carriers in all the carriers awarded with an order depending on the local and in-transit arrival rates and cost distributions.

We observe that when there are no in-transit carriers and the order arrival rate is less than half of the local carrier arrival rate, more than $50 \%$ of the local carriers abandon without an order. Moreover, when the ratio of order arrival and carrier arrival rates, i.e., $\lambda_{0} /\left(\lambda_{1}+\lambda_{2}\right)$, is greater than $60 \%$, more than half of the local carriers receive an order although most of the orders are received by in-transit carriers as the intransit arrival rate increases. Since a second-price auction is used, the difference in cost distribution averages and the variability around the averages do not affect $f_{1}$ and $d_{1}$. Their average values for the range of parameters for different values of $\Delta$ and $k$ are $44 \%$ and $54 \%$ respectively. 


\begin{tabular}{|r|rrrrr|}
\hline$f_{1}$ & \multicolumn{5}{|c|}{$\lambda_{0}$} \\
\hline$\lambda_{2}$ & 0.25 & 0.50 & 1.00 & 1.50 & 2.00 \\
\hline 0.00 & $22 \%$ & $42 \%$ & $70 \%$ & $85 \%$ & $93 \%$ \\
0.50 & $14 \%$ & $29 \%$ & $56 \%$ & $75 \%$ & $86 \%$ \\
1.00 & $9 \%$ & $20 \%$ & $43 \%$ & $63 \%$ & $77 \%$ \\
1.50 & $6 \%$ & $14 \%$ & $32 \%$ & $51 \%$ & $67 \%$ \\
2.00 & $4 \%$ & $10 \%$ & $24 \%$ & $40 \%$ & $56 \%$ \\
\hline
\end{tabular}

\begin{tabular}{|r|rrrrr|}
\hline$d_{1}$ & \multicolumn{5}{|c|}{$\lambda_{0}$} \\
\hline$\lambda_{2}$ & 0.25 & 0.50 & 1.00 & 1.50 & 2.00 \\
\hline 0.00 & $100 \%$ & $100 \%$ & $100 \%$ & $100 \%$ & $100 \%$ \\
0.50 & $60 \%$ & $63 \%$ & $66 \%$ & $67 \%$ & $67 \%$ \\
1.00 & $38 \%$ & $42 \%$ & $47 \%$ & $49 \%$ & $50 \%$ \\
1.50 & $26 \%$ & $29 \%$ & $34 \%$ & $37 \%$ & $39 \%$ \\
2.00 & $18 \%$ & $20 \%$ & $25 \%$ & $28 \%$ & $31 \%$ \\
\hline
\end{tabular}

Table 3. Percentage of local carriers receiving an order and the fraction of local carriers in all the carriers awarded with an order

Table 4 analyzes the effect of carrier arrival rates and cost distributions on the average profits of local carriers. Since a second-price auction is used, a local carrier can win an auction only when there are no intransit carriers in the auction. Therefore, given that a local carrier is awarded with an order, its expected profit is not affected substantially by the in-transit carriers as shown in Table 4 .

\begin{tabular}{|r|rrrrr|}
\hline $\bar{Q}_{1}$ & \multicolumn{5}{|c|}{$\lambda_{0}$} \\
\hline$\lambda_{2}$ & 0.25 & 0.50 & 1.00 & 1.50 & 2.00 \\
\hline 0.00 & 0.153 & 0.164 & 0.181 & 0.190 & 0.195 \\
0.50 & 0.147 & 0.156 & 0.171 & 0.183 & 0.191 \\
1.00 & 0.144 & 0.150 & 0.163 & 0.175 & 0.185 \\
1.50 & 0.142 & 0.147 & 0.157 & 0.168 & 0.178 \\
2.00 & 0.141 & 0.144 & 0.153 & 0.162 & 0.171 \\
\hline
\end{tabular}

\begin{tabular}{|r|rrrr|}
\hline \multicolumn{1}{l|}{$\bar{Q}_{1}$} & \multicolumn{4}{|c|}{$k$} \\
\hline$\Delta$ & 0.50 & 0.60 & 0.70 & 0.80 \\
\hline 0.10 & 0.082 & 0.082 & 0.082 & 0.082 \\
0.20 & 0.164 & 0.164 & 0.164 & 0.164 \\
0.30 & 0.247 & 0.247 & 0.247 & 0.247 \\
\hline
\end{tabular}

Table 4. Expected profit of local carriers

Effect on In-Transit Carriers. Although in-transit carriers have an advantage due to their lower costs, an auction may not be opened during the time they stay at the logistics market. Since their abandonment rates are higher than the local carriers, some of the orders are received by the local carriers that stay longer at the logistics market. Table 5 shows that when $\lambda_{0} /\left(\lambda_{1}+\lambda_{2}\right)$ is less than $40 \%$, more than half of the arriving in-transit carriers leave the logistics market without an order. However when the intransit arrival rate reaches and exceeds the local carrier arrival rate, most of the orders are awarded to intransit carriers.

\begin{tabular}{|r|rrrrr|}
\hline$f_{2}$ & \multicolumn{5}{|c|}{$\lambda_{0}$} \\
\hline$\lambda_{2}$ & 0.25 & 0.50 & 1.00 & 1.50 & 2.00 \\
\hline 0.00 & 0.000 & 0.000 & 0.000 & 0.000 & 0.000 \\
0.50 & 0.189 & 0.338 & 0.567 & 0.734 & 0.845 \\
1.00 & 0.150 & 0.277 & 0.484 & 0.646 & 0.770 \\
1.50 & 0.122 & 0.232 & 0.418 & 0.571 & 0.696 \\
2.00 & 0.102 & 0.197 & 0.365 & 0.507 & 0.629 \\
\hline
\end{tabular}

\begin{tabular}{|r|rrrrr|}
\hline$d_{2}$ & \multicolumn{5}{|c|}{$\lambda_{0}$} \\
\hline$\lambda_{2}$ & 0.25 & 0.50 & 1.00 & 1.50 & 2.00 \\
\hline 0.00 & $0 \%$ & $0 \%$ & $0 \%$ & $0 \%$ & $0 \%$ \\
0.50 & $40 \%$ & $37 \%$ & $34 \%$ & $33 \%$ & $33 \%$ \\
1.00 & $62 \%$ & $58 \%$ & $53 \%$ & $51 \%$ & $50 \%$ \\
1.50 & $74 \%$ & $71 \%$ & $66 \%$ & $63 \%$ & $61 \%$ \\
2.00 & $82 \%$ & $80 \%$ & $75 \%$ & $72 \%$ & $69 \%$ \\
\hline
\end{tabular}

Table 5. Percentage of in-transit carriers receiving an order and the fraction of in-transit carriers in all the carriers awarded with an order

The expected profits of in-transit carriers depend heavily on the ratio of in-transit and local carrier arrival rates. As the in-transit carrier arrival rate increases, the price will decrease by the bids of competing in-transit carriers. Therefore, the expected profit of in-transit carriers is higher when their arrival rate is lower. Since the price is determined by a second price auction, their expected profit increases with the 
difference between the average costs of local and in-transit carriers. Similarly, $f_{2}$ and $d_{2}$ are not affected by $\Delta$ and $k$ and their average values are $35 \%$ and $46 \%$ respectively.

\begin{tabular}{|r|rrrrr|}
\hline $\bar{Q}_{2}$ & \multicolumn{5}{|c|}{$\lambda_{0}$} \\
\hline$\lambda_{2}$ & 0.25 & 0.50 & 1.00 & 1.50 & 2.00 \\
\hline 0.00 & 0.000 & 0.000 & 0.000 & 0.000 & 0.000 \\
0.50 & 0.557 & 0.596 & 0.673 & 0.739 & 0.785 \\
1.00 & 0.451 & 0.490 & 0.569 & 0.648 & 0.715 \\
1.50 & 0.366 & 0.401 & 0.475 & 0.553 & 0.630 \\
2.00 & 0.299 & 0.329 & 0.394 & 0.466 & 0.543 \\
\hline
\end{tabular}

\begin{tabular}{|r|rrrr|}
\hline \multicolumn{1}{l|}{$\bar{Q}_{2}$} & \multicolumn{4}{|c|}{$k$} \\
\hline$\Delta$ & 0.50 & 0.60 & 0.70 & 0.80 \\
\hline 0.10 & 0.314 & 0.369 & 0.424 & 0.479 \\
0.20 & 0.347 & 0.400 & 0.454 & 0.508 \\
0.30 & 0.379 & 0.431 & 0.484 & 0.536 \\
\hline
\end{tabular}

Table 6. Expected profit of in-transit carriers

Effect on Logistics Market. The interaction between carrier and order arrival and abandonment rates affects the expected number of carriers and orders that will be present at the logistics market in the long run. Tables 7 and 8 show the effects of in-transit and order arrival rates and also the effects of order and in-transit carrier abandonment rates on the total expected number of carriers, $\bar{N}_{T}=\bar{N}_{1}+\bar{N}_{2}$, and on the expected number of orders.

As we expected, when $\lambda_{0} /\left(\lambda_{1}+\lambda_{2}\right)$ is low, the expected number of carriers increases and when this ratio is high, the expected number of orders increases. Similarly, as the in-transit carriers abandon more frequently, $\bar{N}_{T}$ decreases while $\bar{N}_{0}$ increases and as the orders abandon more, $\bar{N}_{T}$ increases while $\bar{N}_{0}$ decreases. Cost distributions do not affect the expected carrier and order values.

\begin{tabular}{|c|rrrrr|}
\hline $\bar{N}_{T}$ & \multicolumn{5}{|c|}{$\lambda_{0}$} \\
\hline$\lambda_{2}$ & 0.25 & 0.50 & 1.00 & 1.50 & 2.00 \\
\hline 0.00 & 1.755 & 1.288 & 0.625 & 0.287 & 0.135 \\
0.50 & 2.430 & 1.996 & 1.218 & 0.672 & 0.353 \\
1.00 & 3.060 & 2.672 & 1.888 & 1.211 & 0.721 \\
1.50 & 3.662 & 3.310 & 2.567 & 1.841 & 1.226 \\
2.00 & 4.250 & 3.921 & 3.225 & 2.503 & 1.823 \\
\hline
\end{tabular}

\begin{tabular}{|c|rrrrr|}
\hline $\bar{N}_{0}$ & \multicolumn{5}{|c|}{$\lambda_{0}$} \\
\hline$\lambda_{2}$ & 0.25 & 0.50 & 1.00 & 1.50 & 2.00 \\
\hline 0.00 & 0.051 & 0.161 & 0.625 & 1.410 & 2.398 \\
0.50 & 0.026 & 0.078 & 0.315 & 0.800 & 1.543 \\
1.00 & 0.014 & 0.043 & 0.169 & 0.449 & 0.943 \\
1.50 & 0.009 & 0.025 & 0.097 & 0.259 & 0.568 \\
2.00 & 0.005 & 0.015 & 0.058 & 0.155 & 0.346 \\
\hline
\end{tabular}

Table 7. Expected number of carriers and orders for different values of arrival rates of in-transit carriers and orders

\begin{tabular}{|c|rrrrr|}
\hline $\bar{N}_{T}$ & \multicolumn{5}{|c|}{$\varphi_{2}$} \\
\hline$\varphi_{0}$ & 0.40 & 0.80 & 1.20 & 1.60 & 2.00 \\
\hline 0.20 & 4.126 & 2.050 & 1.366 & 1.026 & 0.823 \\
0.40 & 4.190 & 2.102 & 1.410 & 1.065 & 0.857 \\
0.60 & 4.228 & 2.135 & 1.439 & 1.091 & 0.881 \\
0.80 & 4.255 & 2.159 & 1.461 & 1.110 & 0.898 \\
1.00 & 4.275 & 2.177 & 1.477 & 1.125 & 0.912 \\
\hline
\end{tabular}




\begin{tabular}{|l|rrrrr|}
\hline $\bar{N}_{0}$ & \multicolumn{5}{|c|}{$\varphi_{2}$} \\
\hline$\varphi_{0}$ & 0.40 & 0.80 & 1.20 & 1.60 & 2.00 \\
\hline 0.20 & 0.643 & 0.740 & 0.803 & 0.848 & 0.881 \\
0.40 & 0.359 & 0.433 & 0.484 & 0.520 & 0.549 \\
0.60 & 0.254 & 0.316 & 0.359 & 0.390 & 0.415 \\
0.80 & 0.199 & 0.252 & 0.289 & 0.317 & 0.339 \\
1.00 & 0.164 & 0.210 & 0.244 & 0.269 & 0.288 \\
\hline
\end{tabular}

Table 8. Expected number of carriers and orders for

different values

of arrival rates of in-transit carriers and orders

Summary of Observations. We now summarize our observations and recommendations for the logistics market as follows:

- In order to provide benefit for the firms by decreasing the transportation prices, the logistics market should attract in-transit carriers. However, in order not to hurt the local carriers, a balance between order arrival rate and the total local and in-transit carrier arrival rates should be maintained. It is observed empirically that when $\lambda_{0} /\left(\lambda_{1}+\lambda_{2}\right)$ is greater than $60 \%$, more than half of the local carriers receive an order and their expected profit stays at the same level.

- If the logistics market attracts more orders without increasing the carrier arrival rate, firms will not benefit substantially.

- Another way of increasing competition is through keeping in-transit carriers longer at the logistics market by decreasing their abandonment rate. Note that this can be achieved through service improvements at the logistics center.

- Finally, a possible way of covering the expenses of the logistics market and also controlling the arrival rates of local and in-transit carriers is charging entry fees for arriving carriers. By charging different fees for local and in-transit carriers, a proper balance between order and carrier arrival rates can be sustained.

\subsection{Analytical Results}

In the previous section, we investigated the effects of system parameters on the performance of the logistics market numerically to determine how much each performance measure is affected. In this section, we give our analytical findings related to the structural properties of the model. Specifically, we use stochastic comparisons to prove that as the arrival rates of carriers or the abandonment rate of orders increase, the expected average auction price decreases. Similarly we show that as the order arrival rate or the carrier abandonment rates increase, the expected average auction price increases.

We prove this structural result in two parts. First we show how the random variables for the number of carriers and orders in the steady state are affected by the changes in arrival and abandonment rates. Then, we prove that when the random variables for the number of carriers and orders in the steady state change in a particular way, the average expected auction price obtained by the steady-state distributions of these random variables changes accordingly. We only provide the proofs for the effect of an increasing type 1 arrival rate. The other cases can be proven in a similar way.

\section{Lemma 2:}

- $N_{0}$ is stochastically increasing, $N_{1}$ is stochastically decreasing, and $N_{2}$ is stochastically decreasing in $\lambda_{0}$.

- $N_{0}$ is stochastically decreasing, $N_{1}$ is stochastically increasing, and $N_{2}$ is stochastically increasing in $\lambda_{1}$. 
- $N_{0}$ is stochastically decreasing, $N_{1}$ is stochastically increasing, and $N_{2}$ is stochastically increasing in $\lambda_{2}$.

- $N_{0}$ is stochastically decreasing, $N_{1}$ is stochastically increasing, and $N_{2}$ is stochastically increasing in $\varphi_{0}$.

- $N_{0}$ is stochastically increasing, $N_{1}$ is stochastically decreasing, and $N_{2}$ is stochastically decreasing in $\varphi_{1}$.

- $N_{0}$ is stochastically increasing, $N_{1}$ is stochastically decreasing, and $N_{2}$ is stochastically decreasing in $\varphi_{2}$.

Proof. Proof is given in the Appendix

The above lemma proves that the random variables for the number of carriers and orders that are present at the logistics center in the long run are affected by the changes in the order and carrier arrival and abandonment rates. We next show how the average expected auction price changes with the arrival and abandonment rates.

\section{Lemma 3:}

$\bar{P}_{A}$ is decreasing in $\lambda_{0}, \lambda_{1}$, and in $\varphi_{0}$ and increasing in $\lambda_{0}, \varphi_{1}$, and $\varphi_{2}$.

Proof. Let us compare two systems where system 1 has arrival rate $\lambda_{1}^{(1)}$ and system 2 has arrival rate $\lambda_{1}^{(2)}$ where $\lambda_{1}^{(1)}>\lambda_{2}^{(2)}$. Then Equation (22) gives

$$
\bar{P}_{A}^{(1)}=\frac{\sum_{n_{1}=2}^{K_{1}} p\left(0, n_{1}, 0\right) \pi^{(1)}\left(0, n_{1}, 0\right)+\sum_{n_{2}=2}^{K_{2}} p\left(0,0, n_{2}\right) \pi^{(1)}\left(0,0, n_{2}\right)+\sum_{n_{1}=1}^{K_{1}} \sum_{n_{2}=1}^{K_{2}} p\left(0, n_{1}, n_{2}\right) \pi^{(1)}\left(0, n_{1}, n_{2}\right)}{\sum_{n_{1}=2}^{K_{1}} \pi^{(1)}\left(0, n_{1}, 0\right)+\sum_{n_{2}=2}^{K_{2}} \pi^{(1)}\left(0,0, n_{2}\right)+\sum_{n_{1}=1}^{K_{1}} \sum_{n_{2}=1}^{K_{2}} \pi^{(1)}\left(0, n_{1}, n_{2}\right)} .
$$

Lemma 2 yields $\sum_{n_{1}=2}^{K_{1}} \pi^{(1)}\left(0, n_{1}, 0\right)>\sum_{n_{1}=2}^{K_{1}} \pi^{(2)}\left(0, n_{1}, 0\right), \sum_{n_{2}=2}^{K_{2}} \pi^{(1)}\left(0,0, n_{2}\right)>\sum_{n_{2}=2}^{K_{2}} \pi^{(2)}\left(0,0, n_{2}\right)$, and

$\sum_{n_{1}=1}^{K_{1}} \sum_{n_{2}=1}^{K_{2}} \pi^{(1)}\left(0, n_{1}, n_{2}\right)>\sum_{n_{1}=1}^{K_{1}} \sum_{n_{2}=1}^{K_{2}} \pi^{(2)}\left(0, n_{1}, n_{2}\right)$. Since $p\left(n_{0}, n_{1}, n_{2}\right)$ is a decreasing function in $n_{1}$ and $n_{2}$ as shown in Lemma 1,

$$
\begin{aligned}
& \sum_{n_{1}=2}^{K_{1}} p\left(0, n_{1}, 0\right) \pi^{(1)}\left(0, n_{1}, 0\right)<\sum_{n_{1}=2}^{K_{1}} p\left(0, n_{1}, 0\right) \pi^{(2)}\left(0, n_{1}, 0\right), \\
& \sum_{n_{2}=2}^{K_{2}} p\left(0,0, n_{2}\right) \pi^{(1)}\left(0,0, n_{2}\right)<\sum_{n_{2}=2}^{K_{2}} p\left(0,0, n_{2}\right) \pi^{(2)}\left(0,0, n_{2}\right) \text {, and } \\
& \sum_{n_{1}=1}^{K_{1}} \sum_{n_{2}=1}^{K_{2}} p\left(0, n_{1}, n_{2}\right) \pi^{(1)}\left(0, n_{1}, n_{2}\right)<\sum_{n_{1}=1}^{K_{1}} \sum_{n_{2}=1}^{K_{2}} p\left(0, n_{1}, n_{2}\right) \pi^{(2)}\left(0, n_{1}, n_{2}\right) \text {. As a result } P_{A}^{(1)}<P_{A}^{(2)} . \text {. }
\end{aligned}
$$

These structural results on the monotonicity of the expected auction price as a function of the arrival and departure rates of the system may be of interest in other settings such as internet auctions or procurement auctions where the number of participants varies randomly over time. 


\section{Conclusions}

In this paper, a logistics spot market, referred to as a logistics center, where the orders from a number of shippers with goods to transport to various destinations are matched with two types of carriers through a reverse auction, is analyzed. The paper is motivated by a logistics auction market in Turkey, ESO Logistics Center, where a reverse auction is used to match carriers with shippers. An interesting characteristic of this market is that local carriers compete with in-transit carriers who may be returning to their bases empty after unloading at a previous stop. The main contribution of this study is to develop an analytical model of the logistics market to analyze the effects of implementing such a logistics spot market on three parties involved: firms, local carriers, and in-transit carriers, and also the effects of various system parameters, such as order and carrier arrival and abandonment rates on the performance of the system.

Our analysis show that the effects of cost distributions, arrival rates, and abandonment rates on local and in-transit carriers are quite intricate and one needs to evaluate the performance of the system for possible values of system parameters. This type of analysis is valuable since ESO Logistics Center has been established relatively recently and is certain to observe growth in traffic volumes in the future. Our analysis may then yield useful insights on future strategic and tactical decisions. Continuing the on-going collaboration with the ESO Logistics Center, the performance evaluation tool should be useful in further development of the auction market and in its management.

In addition, even though our motivation stems from an application in a logistics auction, our modeling approach and results can be applied to repeated auctions in other contexts such as internet auctions or procurement auctions where the number of participants varies randomly over time. As such, our results on the monotonicity of the expected auction price as a function of the arrival and departure rates of the system may be of interest in other settings.

\section{Acknowledgments}

We thank Eskişehir Chamber of Industry (ESO) and ESO Logistics Center for their support in this project. This research is partially supported by TÜBA-GEBİP Program and TÜBİTAK.

\section{References}

Ağrall, S. (2005), Modeling and Analysis of an Auction-Based Logistics Market, M.S. Thesis, Koç University, Industrial Engineering, Istanbul, Turkey.

Alp, O., N.K. Erkip, and R. Güllü (2003), "Outsourcing Logistics: Designing Transportation Contracts between a Manufacturer and a Transporter," Transportation Science, Vol. 37, pp. 23-39.

Bhattacharya, P.P. and A. Ephremides (1991), "Stochastic Monotonicity Properties of Multiserver Queues with Impatient Customers," Journal of Applied Probability, Vol. 28, pp. 673-682.

Chen, R.R., R. Roundy, and G. Janakiraman, R.Q. Zhang (2005), "Efficient Auction Mechanisms for Supply Chain Procurement," Management Science, Vol. 51, No. 3, pp. 467-482. 
Elmaghraby, W. (2000), "Supply contract competition and sourcing policies," Manufacturing and Service Operations Management, Vol. 2, pp. 350-371.

Klemperer, P. (1999), “Auction Theory: A Guide to the Literature," Journal of Economic Survey, Vol. 13, pp. 227-278.

Ledyard, J.L, M. Olson, D. Porter, J. A. Swanson, and D. P.Torma (2002), “The First Use of a Combined Value Auction for Transportation Services," Interfaces, Vol. 32, No. 5, pp. 4-12.

Milgrom, P. (1989), “Auctions and Bidding: A Primer, Journal of Economic Perspectives," Vol. 3, pp. 3-22.

Nandiraju, S. and A. Regan (2003), Freight Transportation Electronic Marketplaces: A Survey of the Industry and Exploration of Important Research Areas, Tecbnical report: UCI-ITS-LI-03-12, Institute of Transportation Studies, University of California, Irvine.

Nandula, M. and S.P. Dutta (2000), "Performance Evaluation of an Auction-Based Manufacturing System Using Colored-Petri Nets," International Journal of Production Research, Vol. 38, pp. 2155-2171.

Qi, M. and M. Pich (2002), CargoExchange.Net Pte Ltd, Case Study, INSEAD.

Segev, A., C. Beam, and G. Shanthikumar (2001), "Optimal Design of Internet-Based Auctions," Information Technology and Management, Vol. 2, pp.121-163.

Shaked, M. and J.G. Shanthikumar (1994), Stochastic Orders and Their Applications, Academic Press.

Song, J. and A. Regan (2003), An Auction Based Collaborative Carrier Network, Technical report: UCI-ITSWP-03-6, Institute of Transportation Studies, University of California, Irvine.

Vakrat, Y. and A. Seidmann (2000), "Implications of the Bidders' Arrival Process on the Design of Online Auctions," Proceedings of the $33^{\text {rd }}$ Hawaii International Conference on System Sciences.

Veeramani, D. and K. Wang (1997), "Performance Analysis of Auction-Based Distributed Shop-Floor Control Schemes from the Perspective of the Communication System," The International Journal of Flexible Manufacturing Systems, Vol. 9, pp. 121-143.

Vickrey, W. (1961), "Counterspeculation, Auctions and Competitive Sealed Tenders," Journal of Finance, Vol. 16, pp. 8-37. 


\section{Appendix}

Summary of the Information Available in the ESO Database:

The data set received from ESO Logistics Center includes detailed information about orders and bids received between December 2003 and May 2005. During this period, 1717 transportation orders that were opened by shippers for transportation from Eskişehir to 44 different cities and 1549 of these orders received bids from carriers and 168 orders were cancelled (abandoned). Table 9 reports all the orders and bids.

\begin{tabular}{|c|c|c|c|c|c|c|c|c|}
\hline City & $\begin{array}{c}\text { Average } \\
\text { price (YTL) }\end{array}$ & $\begin{array}{c}\text { Average bid } \\
\text { (YTL) }\end{array}$ & $\begin{array}{c}\text { Total } \\
\text { number of } \\
\text { orders }\end{array}$ & $\begin{array}{c}\text { Total } \\
\text { number of } \\
\text { realized } \\
\text { orders }\end{array}$ & $\begin{array}{c}\text { Total number of } \\
\text { orders that carriers } \\
\text { give bids }\end{array}$ & $\begin{array}{c}\text { Total } \\
\text { number of } \\
\text { bids }\end{array}$ & $\begin{array}{c}\text { Average } \\
\text { number of } \\
\text { bids for each } \\
\text { demand }\end{array}$ & Distance $(\mathrm{km})$ \\
\hline 1 & 354 & 357 & 185 & 97 & 130 & 302 & 2.32 & 688 \\
\hline 3 & 128 & 135 & 11 & 6 & 8 & 10 & 1.25 & 144 \\
\hline 5 & 340 & 365 & 52 & 26 & 38 & 42 & 1.11 & 569 \\
\hline 6 & 185 & 211 & 51 & 6 & 11 & 23 & 2.09 & 233 \\
\hline 7 & 400 & 466 & 69 & 2 & 12 & 6 & 0.50 & 428 \\
\hline 9 & 390 & 482 & 18 & 2 & 7 & 5 & 0.71 & 487 \\
\hline 11 & 50 & 76 & 11 & 1 & 3 & 3 & 1.00 & 80 \\
\hline 15 & 354 & 354 & 1 & 1 & 1 & 1 & 1.00 & 306 \\
\hline 16 & 103 & 160 & 27 & 4 & 10 & 17 & 1.70 & 149 \\
\hline 17 & 311 & 311 & 10 & 3 & 4 & 5 & 1.25 & 421 \\
\hline 25 & 1150 & 1785 & 10 & 1 & 2 & 5 & 2.50 & 1109 \\
\hline 26 & 95 & 146 & 2 & 2 & 2 & 4 & 2.00 & 0 \\
\hline 27 & 504 & 544 & 80 & 32 & 49 & 85 & 1.73 & 894 \\
\hline 34 & 216 & 227 & 257 & 78 & 108 & 4 & 0.04 & 330 \\
\hline 35 & 238 & 255 & 90 & 49 & 60 & 119 & 1.98 & 412 \\
\hline 41 & 181 & 185 & 393 & 176 & 223 & 306 & 1.37 & 219 \\
\hline 42 & 345 & 375 & 22 & 3 & 3 & 9 & 3.00 & 338 \\
\hline 43 & 90 & 95 & 4 & 1 & 2 & 2 & 1.00 & 78 \\
\hline 44 & 675 & 775 & 16 & 1 & 5 & 4 & 0.80 & 883 \\
\hline 45 & 225 & 233 & 13 & 6 & 7 & 15 & 2.14 & 394 \\
\hline 48 & 415 & 565 & 64 & 1 & 12 & 11 & 0.92 & 506 \\
\hline 59 & 283 & 301 & 28 & 14 & 19 & 48 & 2.53 & 462 \\
\hline 63 & 700 & 700 & 1 & 1 & 1 & 2 & 2.00 & 1031 \\
\hline 64 & 192 & 196 & 59 & 29 & 34 & 52 & 1.53 & 219 \\
\hline 81 & 190 & 206 & 75 & 27 & 35 & 46 & 1.31 & 251 \\
\hline Overall & 324.56 & 380.20 & 1549 & 569 & 786 & 1126 & 1.51 & 425.24 \\
\hline
\end{tabular}

Table 9. Summary information of ESO auction database 


\section{Proof of Lemma 1:}

Let us consider the first order difference of the expected auction price $p\left(n_{0}, n_{1}, n_{2}\right)$ in $n_{2}$ : $p\left(n_{0}, n_{1}, n_{2}\right)-p\left(n_{0}, n_{1}, n_{2}+1\right)$. First, let us consider the case $n_{2}>1$. Equation (2) gives

$$
p\left(0, n_{1}, n_{2}\right)-p\left(0, n_{1}, n_{2}+1\right)=\int_{c_{2}}^{c_{2}^{\prime}}\left[1-F_{2}(x)\right]^{n_{2}-1} n_{2} F_{2}^{2}(x) d x \geq 0 .
$$

Let us now consider the case $n_{1}>0, n_{2}=1$. In this case, the type 2 carrier gets the order and he is paid at a price which is the lowest cost among the costs of $n_{1}$ type 1 carriers. When there is one additional type 2 carrier, i.e, $n_{1}>0, n_{2}=2$, then one of the type 2 carriers that has a lower cost gets the order and he is paid at the second lowest bid which is the cost of the other type 2 carrier. Since the cost distributions of type 1 and type 2 carriers are disjoint and $c_{1}>c_{2}^{\prime}$, then $p\left(0, n_{1}, 1\right)>p\left(0, n_{1}, 2\right), n_{1} \geq 0$.

When $n_{1}=0$ and $n_{2}=1, p(0,0,1)=c_{1}^{\prime}$. If $n_{1}=0$ and $n_{2}=2$, then $p(0,0,2)$ is the expected minimum of the costs of two type 2 carriers. Since $F_{2}(x) \leq c_{1}^{\prime}, \forall x, p(0,0,1) \geq p(0,0,2)$. Similarly $p(0,1,0)=c_{1}^{\prime}$ and $p(0,1$, $1)$ is equal to the cost of the type 2 carrier. Since $F_{2}(x) \leq c_{1}^{\prime}, \forall x, p(0,1,0,0) \geq p(0,1,1,0)$. Finally, when $n_{1}>1$ and $n_{2}=0$,

$$
p\left(0, n_{1}, 0\right)-p\left(0, n_{1}, 1\right)=\int_{c_{1}}^{c_{1}^{\prime}} n_{1}\left[1-F_{1}(x)\right]^{n_{1}-1} F_{1}(x) d x \geq 0
$$

As a result, $p\left(n_{0}, n_{1}, n_{2}\right)-p\left(n_{0}, n_{1}, n_{2}+1\right)>0$ and $p(l, b, s)$ is non-increasing in $n_{2}$.

Similarly, let us consider the first order difference of the expected auction price $p\left(n_{0}, n_{1}, n_{2}\right)$ in $n_{1}$ : $p\left(n_{0}, n_{1}, n_{2}\right)-p\left(n_{0}, n_{1}+1, n_{2}\right)$. When $n_{2}>1, p\left(0, n_{1}, n_{2}\right)-p\left(0, n_{1}+1, n_{2}\right)=0$. In words, if there are at least two type 2 carriers, the number of type 1 carriers does not affect the average expected auction price. When $n_{2}=1$ and $n_{1}>0$, Equation (2) yields

$$
p\left(0, n_{1}, 1\right)-p\left(0, n_{1}+1,1\right)=\int_{c_{1}}^{c_{1}^{\prime}}\left[1-F_{1}(x)\right]^{n_{1}} F_{1}(x) d x \geq 0 .
$$

When $n_{2}=0$ and $n_{1}>1$, it can be shown by using Equation (2) that

$$
p\left(0, n_{1}, 0\right)-p\left(0, n_{1}+1,0\right)=\int_{c_{1}}^{c_{1}^{\prime}} n_{1}\left[1-F_{1}(x)\right]^{n_{1}-1} F_{1}^{2}(x) d x \geq 0 \text {. }
$$

Note that $p(0,1,0)=c_{1}^{\prime}$ and $p(0,2,0)$ is the expected minimum of the costs of two type 1 carriers. Since $F_{1}(x) \leq c_{1}^{\prime}$ for all $x, p(0,1,0) \geq p(0,2,0)$. Similarly, $p(0,0,1)=c_{1}^{\prime}$ and $p(0,1,1)$ is equal to the cost of the type 2 carrier. Since $F_{2}(x) \leq c_{1}^{\prime}$ for all $x, p(0,0,1) \geq p(0,1,1)$.

As a result, $p\left(n_{0}, n_{1}, n_{2}\right)-p\left(n_{0}, n_{1}+1, n_{2}\right) \geq 0$ and $p\left(n_{0}, n_{1}, n_{2}\right)$ is non-increasing in $n_{1}$.

\section{Proof of Lemma 2:}

We use a standard sample-path coupling argument (see Shaked and Shanthikumar (1994) for example). As usual, a uniformization of the continuous-time process facilitates the comparisons. To this end, let $\gamma=\lambda_{0}+\lambda_{1}+\lambda_{2}+K_{0} \varphi_{0}+K_{1} \varphi_{1}+K_{2} \varphi_{2}$. In the uniformized chain a transition takes place at exponentially 
distributed intervals with rate $\gamma$, but some of these transitions may be fictitious and may not change the system state.

Let us compare two systems where system 1 has arrival rate $\lambda_{1}^{(1)}$ and system 2 has arrival rate $\lambda_{1}^{(2)}$ where $\lambda_{1}^{(1)}>\lambda_{1}^{(2)}$. Assume that we compare systems 1 and 2 starting from an empty state: $\left(N_{0}^{(1)}(0), N_{1}^{(1)}(0), N_{2}^{(1)}(0)\right)=\left(N_{0}^{(2)}(0), N_{1}^{(2)}(0), N_{2}^{(2)}(0)\right)=(0,0,0)$, and let us also assume that at the time of the $n$th transition $\left(N_{0}^{(1)}\left(t_{n}\right), N_{1}^{(1)}\left(t_{n}\right), N_{2}^{(1)}\left(t_{n}\right)\right)=(z, x, y)$ and $\left(N_{0}^{(2)}\left(t_{n}\right), N_{1}^{(2)}\left(t_{n}\right), N_{2}^{(2)}\left(t_{n}\right)\right)=\left(z^{\prime}, x^{\prime}, y^{\prime}\right)$ where $x \geq x^{\prime}, y \geq y^{\prime}$, and $z \leq z^{\prime}$. We then argue that, the next transition will preserve the same order for each component. In order to do that, we look at the effect of each transition in both systems.

i. A type 1 - arrival: This event can lead to three different states starting from the state $(z, x, y)$. Let us number the different transition types as follows:

1. to state $(z, x+1, y)$ if $x<K_{1}$ and $z=0$

2. to state $(z, x, y) \quad$ if $x=K_{1}$ and $z=0$

3. to state $(z-1,0,0)$ if $x=0, y=0$, and $z \geq 1$

In order to establish the comparison between the two systems, we need to argue that all possible transition pairs in systems 1 and 2 preserve the orders. This requires checking 9 ( 3 by 3 ) possible transition pairs. Fortunately, most of the pairs are trivially verified. To outline the procedure let us take a type 1 transition for both systems. Then the next state for the two systems are respectively: $(z, x+1, y)$ and $\left(z^{\prime}, x^{\prime}+1, y^{\prime}\right)$, verifying the required inequality: $x+1 \geq x^{\prime}+1, y \geq y^{\prime}$, and $z \leq z^{\prime}$. A more interesting comparison occurs when system 1 makes a type 2 transition and system 2 makes a type 1 transition. The next states are respectively: $(z, x, y)$ and $\left(z^{\prime}, x^{\prime}+1, y\right)$ but recall that a type 2 transition only occurs when $x=K_{1}$. This implies that $x^{\prime}+1$ cannot exceed $x$ with this transition. Another non-trivial comparison is that of a transition 3 in system 1 versus a transition 1 in system 2 . A transition 3 in system 1 implies that $x=0$ and $y=0$, and $z \geq 0$. By the induction assumption, this in turn requires that $x^{\prime}=0$ and $y^{\prime}=0$, and $z^{>}>z$, therefore a type 1 transition is impossible in system 2. We summarize the results in the below table, whose entries $(i, j)$ display how the induction hypothesis is preserved for transition $i$ in system 1 and transition $j$ in system 2 .

\begin{tabular}{|c|c|c|c|}
\hline System $1 \backslash$ System 2 & Transition 1 & Transition 2 & Transition 3 \\
\hline Transition 1 & Follows directly & Follows directly & Follows directly \\
\hline Transition 2 & Verified & Follows directly & Follows directly \\
\hline Transition 3 & Impossible & Impossible & Follows directly \\
\hline
\end{tabular}

Finally, note that due to coupling, and to the fact that $\lambda_{1}^{(1)}>\lambda_{1}^{(2)}$ there may be cases where a type $1-$ arrival to system is a real arrival to system 1 but only a fictitious arrival to system 2 . In this case, system 2 does not change state whereas system 1 can go to either state $(z, x+1, y)$ or to state $(z-1,0,0)$ preserving the required inequality in both cases.

ii. A type 2 arrival: This event takes system 1 to three different states. Let us number the different transition types as follows:

1. to state $(z, x, y+1)$ if $y<K_{2}$ and $z=0$ 
2. to state $(z, x, y) \quad$ if $y=K_{2}$ and $z=0$

3. to state $(z-1,0,0)$ if $x=0, y=0$, and $z \geq 1$

This case is very similar to case $i$. The results can be summarized in the below table:

\begin{tabular}{|c|c|c|c|}
\hline System $1 \backslash$ System 2 & Transition 1 & Transition 2 & Transition 3 \\
\hline Transition 1 & Follows directly & Follows directly & Follows directly \\
\hline Transition 2 & Verified & Follows directly & Follows directly \\
\hline Transition 3 & Impossible & Impossible & Follows directly \\
\hline
\end{tabular}

iii. An order arrival: This event takes system 1 to four different states. Let us number the different transition types as follows:
1. to state $\left(z, x_{2} y-1\right)$ if $y>0$ and $z=0$
2. to state $(z, x-1, y)$ if $x>0, y=0$, and $z=0$
3. to state $\left(z^{+1,0,0)}\right.$ if $x=0, y=0$, and $z^{<}<K_{0}$
4. to state $(z, 0,0) \quad$ if $x=0, y=0$, and $z=K_{0}$

We summarize the results in the following table. A non-trivial comparison occurs when system 1 makes transition 1 and system 2 makes transition 2. However, this transition implies $y>y$ ' and hence at the next transition $y-1 \geq y^{\prime}$.

\begin{tabular}{|c|c|c|c|c|}
\hline System $1 \backslash$ System 2 & Transition 1 & Transition 2 & Transition 3 & Transition 4 \\
\hline Transition 1 & Follows directly & Verified & Follows directly & Follows directly \\
\hline Transition 2 & Impossible & Follows directly & Follows directly & Follows directly \\
\hline Transition 3 & Impossible & Impossible & Follows directly & Follows directly \\
\hline Transition 4 & Impossible & Impossible & Impossible & Follows directly \\
\hline
\end{tabular}

iv. A Type 1 abandonment: This event takes system 1 to two different states. Let us number the different transition types as follows:

$$
\begin{array}{ll}
\text { 1. to state }(z, x-1, y) & \text { if } x>0 \text { and } z=0 \\
\text { 2. to state }(z, x, y) & \text { if } x=0 \text { and } z=0
\end{array}
$$

This event is somewhat different than the first three events considered since the actual transition rates are state dependent. According to our coupling argument, a real transition (abandonment) at state $\left(z, x_{2} y\right)$ in system 1 may correspond to a fictitious transition for system 2 at state $\left(z^{\prime}, x^{\prime}, y\right)$ (when $x>x$ ) (in fact, this happens with probability $\left.x / x^{\prime}\right)$ whereas a real transition (abandonment) at state ( $\left.z^{\prime}, x^{\prime}, y^{\prime}\right)$ in system 2 always corresponds to a real transition at state $(z, x, y)$ in system 1 (where $x^{2}>x$ and $x^{2}>0$ ). Now let us consider the different pairs of transitions in systems 1 and 2 . A real transition in system 1 leads to the state $(z, x-1, y)$ or $(z, x, y)$ but the corresponding transition in system 2 can be fictitious only if $x>x^{\prime}$ which ensures that $x-1 \geq x^{2}$. Otherwise checking the pairs we find that transition 2 in system 1 and transition 1 in system 2 is impossible since $x \geq x^{\prime}$ by the induction assumption. The other combinations are easily verified.

v. A Type 2 abandonment: This event takes the system 1 to two different states. Let us number the different transition types as follows:
1. to state $(z, x, y-1)$
if $y>0$ and $z=0$
2. to state $(z, x, y)$
if $y=0$ and $z=0$ 
This case is very similar to case 4 and parallel arguments apply.

vi. An order abandonment: This event takes the system 1 to two different states. Let us number the different transition types as follows:

1. to state $(z-1, x, y)$ if $z>0$

2. to state $(z, x, y)$ if $z=0$

Using the coupling argument, since $z \geq z$, there may be actual transitions (order abandonments) in system 2 with corresponding fictitious transitions in system 1. This does not oppose the induction assumption since this can happen only when $z>₹$ which implies that after the state transition we still have $z-1 \geq z$. The other pairs of transitions are also directly verified.

We have hence proven that for the coupled system $N_{0}^{(1)}\left(t_{n}\right) \leq N_{0}^{(2)}\left(t_{n}\right), N_{1}^{(1)}\left(t_{n}\right) \geq N_{1}^{(2)}\left(t_{n}\right)$ and $N_{2}^{(1)}\left(t_{n}\right) \geq N_{2}^{(2)}\left(t_{n}\right)$ and for all transition times $t_{n}$. Now passing to the corresponding stationary random variables $N_{0}^{(1)}, N_{0}^{(2)}, N_{1}^{(1)}, N_{1}^{(2)}, N_{2}^{(1)}, N_{2}^{(2)}$, we immediately obtain the desired stochastic comparison:

$$
\left(N_{0}^{(2)}, N_{1}^{(1)}, N_{2}^{(1)}\right) \geq_{s t}\left(N_{0}^{(1)}, N_{1}^{(2)}, N_{2}^{(2)}\right)
$$

or equivalently, $\operatorname{Prob}\left[N_{0}^{(1)} \leq z, N_{1}^{(1)}>x, N_{2}^{(1)}>y,\right] \geq \operatorname{Prob}\left[N_{0}^{(2)} \leq z, N_{1}^{(2)}>x, N_{2}^{(2)}>y\right]$. 。 